$\begin{array}{cl}\begin{array}{c}\text { Revue } \\ \text { de Ihistoire }\end{array} & \text { Revue de l'histoire des religions } \\ \text { des religions } & 2 \mid 2005 \\ & \text { Varia }\end{array}$

\title{
Propos sur le feu au pays du vodu Un pont entre hommes et dieux en Afrique
}

Words about fire in the land of the Vodu. A link between Man and Gods in Africa

Alain Macé

\section{OpenEdition}

Journals

Édition électronique

URL : http://journals.openedition.org/rhr/4181

DOI : $10.4000 /$ rhr.4181

ISSN : 2105-2573

Éditeur

Armand Colin

Édition imprimée

Date de publication : 1 avril 2005

Pagination : 131-176

ISBN : 2200-9285-2

ISSN : 0035-1423

Référence électronique

Alain Macé, «Propos sur le feu au pays du vodu Un pont entre hommes et dieux en Afrique ", Revue de I'histoire des religions [En ligne], 2 | 2005, mis en ligne le 22 janvier 2010, consulté le 03 mai 2019. URL: http://journals.openedition.org/rhr/4181; DOI : 10.4000/rhr.4181 


\section{Propos sur le feu au pays du vodu Un pont entre hommes et dieux en Afrique}

Les études ayant trait à la religion ancestrale sur l'ancienne Côte des Esclaves n'ont guère retenu la présence d'un facteur énergétique, dont useraient les puissances surnaturelles. Le feu y joue pourtant un rôle central, comme dans la sphère du profane. Les Ewé, par exemple, voient à travers le feu (dzo) une clef de lecture du monde et de divers aspects du social. Naît ainsi un discours dont la visée éthique est nette, même s'il reste difficile de repérer les éventuels archaïsmes de son volet religieux.

\section{Words about fire in the land of the Vodu.}

\section{A link between Man and Gods in Africa}

As far as the ancestral religion on the ancient Slave Coast, studies didn't pay attention to an energising factor the supernatural forces might use. Fire is however central, and in the profane world too. For instance, Ewe people consider fire (dzo) as an essential element to examine individual relationship with the environment and society. Hence rises a discourse in which one the ethical aim is patent, even if it is still difficult to locate the eventual archaisms of its religious dimension. 
Les pratiques religieuses des populations de l'ancienne Côte des Esclaves (Ghana oriental, Togo, Bénin et Nigeria occidental) font l'objet d'observations et de commentaires depuis le début du $\mathrm{XVI}^{\mathrm{e}}$ siècle. Après des comptes rendus de voyages hauts en couleur ${ }^{1}$ durant la période pré-coloniale, naissent ensuite des orientations soucieuses d'appréhender les rapports au sacré sous un angle désormais scientifique. Parmi de très nombreuses études, citons celles sur les cosmogonies de L. Frobenius ${ }^{2}$, de P. F. Verger ${ }^{3}$ et de H. Courlander ${ }^{4}$, les expressions du sacré de J. Spieth ${ }^{5}$, A. Le Hérissé ${ }^{6}$, M. J. Herskovits ${ }^{7}$, C. Rivière ${ }^{8}$ et A. de Surgy ${ }^{9}$, la

1. Duarte Pacheco Pereira, Esmeraldo de Situ Orbis 1505-1506, traduction de R. Mauny, Bissau, 1956. Parmi les découvreurs de son époque, D. Pacheco Pereira se distingue par un propos laconique sur le religieux. Son apport se limite à la mention de «nègres idolâtres » et de «fétiches » (avec la connotation péjorative d'alors). Voir aussi: Pieter de Marees, Description et récit historial du royaume d'or de Guinée, (trad. fr.), Amsterdam, 1605 ; Guillaume Bosman, Voyage de Guinée, (trad. fr.), Utrecht, 1705 ; Jean-Baptiste Labat, Voyage du chevalier des Marchais en Guinée, isles voisines et à Cayenne, Paris, 1730.

2. Léo Frobenius, The Voice of Africa, London, 1912 et Mythologie de l'Atlantide. Le Poseidon de l'Afrique noire, son culte chez les Yoruba du Bénin, (trad. fr.), Paris, Gallimard, 1949.

3. Pierre Fatumbi Verger, Notes sur le culte des Orisa et Vodun à Bahia, la baie de tous les saints, au Brésil et à l'ancienne Côte des Esclaves en Afrique, Dakar, I.F.A.N, 1957. Les dieux yoruba en Afrique et au Nouveau Monde, Paris, Métaillé, 1982.

4. Harold Courlander, Tales of Yoruba Gods and Heroes. Myths, Legends and Heroic tales of the Yoruba people of West Africa, New York, Crown Publishers, 1973.

5. Jakob Spieth, Die Ewe-Stämme. Material zur Kunde des Ewe Volkes in Deutsch-Togo, Berlin, Diedrich Reimer, 1906. Die Religion der Eweer in SüdTogo, Göttingen, Vandenhoeck u. Ruprecht, 1911.

6. Auguste Le Hérissé, L'Ancien Royaume du Dahomey. Mæurs, religion, histoire, Paris, Larose, 1911.

7. Melville Jean Herskovits, Dahomey an Ancient West African Kingdom, vol I and II, Evanston, Northwestern University Press, 1967.

8. Claude Rivière, Anthropologie religieuse des Evé du Togo, Lomé, Les Nouvelles Editions Africaines, 1981.

9. Albert de Surgy, Le système religieux des Evhé, Paris, L'Harmattan, 1988. 
divination de B. Maupoil ${ }^{10}$ et A. de Surgy ${ }^{11}$, ainsi que celles sur les figures d'identification (le héros, le dieu, l'ancêtre) et les rituels de M. Augé ${ }^{12}$. Il arrive aussi de voir s'instaurer un dialogue entre spiritualité, philosophie et science ${ }^{13}$. Sauf ici ou là à propos du sang des sacrifices, ces remarquables travaux ne s'arrêtent guère sur ce que j'appellerai « le jeu sur les flux d'énergie ». Au mieux J. Spieth parle de « la fraîcheur opposée à la chaleur » ${ }^{14}, \mathrm{~B}$. Maupoil évoque « la flamme divine » ${ }^{15}$, P. F. Verger ${ }^{16}$ voit à travers le culte des orisha ${ }^{17}$ la recherche d'une maîtrise des forces de la nature, M. Augé établit une analogie entre la « force » des vodu et l' « énergie électrique ou atomique ${ }^{18}$ et, enfin, A. de Surgy élabore la notion « d'énergie spirituelle » ${ }^{19}$ après avoir avancé que les charmes recèleraient une «formule concrète d'énergie vitale ${ }^{20}$. A. de Surgy est indubitablement le premier à entreprendre une réflexion sur le thème d'un facteur énergétique incontournable : « [L'énergie spirituelle] appartient à une vie préexistante aux corps, propre à induire ou superviser la formation de corps, et non à celle manifestée automatiquement après coup par ceux-ci en raison du bel arrangement

10. Bernard Maupoil, La Géomancie à l'ancienne côte des esclaves, Paris, Institut d'Ethnologie, 1943.

11. A. de Surgy, La géomancie et le culte d'Afa chez les Evhé du littoral, Paris, Publications Orientalistes de France, 1981.

12. Marc Augé, Pouvoirs de vie, pouvoirs de mort, Paris, Flammarion, 1977 ; Génie du paganisme, Paris, Gallimard, 1982 et Le dieu objet, Paris, Flammarion, 1988 .

13. A. de Surgy, La voie des fétiches. Essai sur le fondement théorique et la perspective mystique des pratiques des féticheurs, Paris, L'Harmattan, 1995.

14. Op. cit., Ch. III, p. 222, n² 1911.

15. Op. cit., p. 61.

16. Op. cit., p. 11, 1957.

17. Nous nous efforcerons de respecter la transcription utilisée par les auteurs pour les termes vernaculaires dans les citations. Dans tous les autres cas, nous adopterons les conventions les plus fréquemment en usage pour les sons particuliers : $\underline{\mathrm{e}}$ pour [e:], $\underline{\mathrm{i}}$ pour [i:], o pour [œ], $\underline{\mathrm{n}}$ pour [gn], $\underline{\mathrm{f}}$ pour [ph], $\underline{\mathrm{d}}$ pour $[\mathrm{dh}], \underline{\mathrm{s}}$ pour $[\mathrm{sh}]$ et $\mathrm{y}$ pour $[\mathrm{hw}]$. Les accents marqueront les tons (hauts, bas, montants puis descendants).

18. Op. cit., p. 23, 94, 1988.

19. Op. cit., p. 91-97, 1995.

20. A. de Surgy, «Les ingrédients des fétiches », Système de pensée en Afrique noire, 12, p. 103-143, 1993. 
de leurs parties.${ }^{21} »$ Hélas, sauf pour la qualifier de « spirituelle » (du latin spiritus, souffle, précise A. de Surgy) et lui conférer une antériorité absolue, ce discours nous renseigne peu sur cette énergie. Il est donc nécessaire de se tourner vers des travaux encore proches du terrain et, surtout, de se rendre à notre tour sur le terrain.

Revenons d'abord aux investigations de P. F. Verger. En discernant la recherche d'une maîtrise des forces de la nature à travers le culte des orisha et des vodu, il émet implicitement l'hypothèse que ceux-ci détiendraient une capacité particulière leur permettant d'agir sur le monde physique. Quoique notre propos risque de s'y heurter, laissons pour l'heure de côté la très difficile question de savoir si les pratiques actuelles se sont superposées « à des croyances plus anciennes d'un caractère différent et plus directement liées aux forces de la nature ${ }^{22}$ et interrogeons-nous plutôt sur cette capacité du surnaturel.

S'il ne faut certes pas exclure d'emblée la présence d'un socle de lointains schémas, la lecture attentive des sources suggère pourtant la prudence : les cultes se rapportent à la nécessité d'intervenir, pour l'essentiel, sur un élément considéré comme profane, à savoir le feu ; la source manifeste des perturbations susceptibles - l'étude lexicographique de M. Delafosse l'a montrée ${ }^{23}$ - d'affecter jusqu'à la vie des hommes ; à l'inverse de la fraîcheur, par définition apaisante et consolatrice ${ }^{24}$. De surcroît de nouvelles observations permettent de franchir une étape en considérant, cette fois-ci sur un mode élargi, le religieux dans ses multiples relations avec un système global de pensée. Pour les Yoruba, les Fon ou encore les Ewé (les trois principaux groupes de la région, culturellement apparentés), le feu n'est pas une simple donnée, un paramètre parmi d'autres, il est l'agent de la dynamique du monde.

21. Op. cit., p. 91, 1995.

22. Op. cit., p. 11, 1957.

23. Maurice Delafosse, Manuel Dahoméen. Grammaire, chrestomathie, dictionnaire français-dahoméen et dahoméen-français, Paris, Leroux, p. 214, 249, 275, 1894.

24. Idem, p. 378-379. 
On observe la quête incessante d'un équilibre idéal entre chaleur et fraîcheur - du reste non exclusivement à l'occasion des rituels mais, de près ou de loin, toujours liée aux interventions du numineux. Une facette non négligeable - essentielle même sous divers aspects - de la perspective holiste (chère aux populations) appelle par conséquent une enquête complémentaire. Ce sera notre hypothèse, le feu occupe le centre du dispositif symbolique à cause justement du rôle fondamental qu'il semble jouer de manière constante - et peut-être même en dehors d'une quelconque considération métaphysique initiale. Cette aire culturelle prête effectivement aux esprits (trowo, vodu, orisha...), la faculté d'intervenir grâce à son emploi (sous forme magique), dont, comme la matière, ils seraient dotés eux aussi. En d'autres termes, sans cet élément censé conférer à toute chose son existence, le surnaturel serait privé de la source d'énergie indispensable à ses manifestations. Corollaire immédiat de cet axiome fondamental, la nature n'est pas une discursivité des esprits en dépit du fait que le pouvoir de l'investir leur soit reconnu.

Entre l'embouchure de la Volta et celle du Mono, notre terrain de 1993 à $1999^{25}$, les Ewé reconnaissent à chaque esprit ${ }^{26}$ la capacité de s'immiscer dans le cours du monde sublunaire au moyen de son feu propre : dzositro $\left(d z o\right.$, le «feu » de $s i^{27}$ et tro $\underline{\text { f }}$ l' « esprit »). À l'échelle de l'individu, l' « attaque » d'une puissance (la « morsure » de son feu) se traduirait d'abord par une subite altération de son état général (perte de poids, insomnies...) ou par une attitude devenue ostensiblement déviante (insultes, menaces, irritabilité...), deux cas de figure requérant des thérapies ou des rituels visant le retour à la «fraîcheur » (fàfà) synonyme de bien-être et de paix. Bien sûr

25. Cette enquête vient en complément d'une autre centrée sur le façonnage du fer en pays éwé. Née de remarques sur le feu en dehors des activités métallurgiques proprement dites, elle fut conduite en majeure partie dans la préfecture du Zio (Togo). Les incursions chez les Ewé du Ghana (autour de Ho et de Kpando) ont permis quant à elles de collecter des informations complémentaires et de procéder à des recoupements.

26. On désignera par « esprits » aussi bien les « esprits errants», que les « âmes de mauvais morts », les « esprits des ancêtres » (trowo $\underline{o}$, sing tró) ou encore les vodu, bref l'ensemble des puissances surnaturelles.

27. M. Augé (op. cit., p. 99, 1988) le précise, la catégorie «si» oscille entre « dépendant de », « complémentaire de » et « femme de ». 
tous les événements ne prennent pas un caractère dramatique, ni n'imposent de prendre sans tarder des mesures afin d'éloigner le spectre d'une rupture du lien social affectant de proche en proche l'ensemble de la communauté. Mais, puisqu'ils surprennent, on soupçonne également l'action d'un feu : la chance, un succès inattendu... auraient toujours, de quelque manière (exigence de sens oblige), une cause bien précise à localiser, si nécessaire au-delà du visible. Face à l'inexplicable de prime abord, les Ewé mobilisent une grille de lecture articulée autour du feu ( $d z o$ ) et de la chaleur ( $d z o d z o$ ), son continuum, un élément et une grandeur par nature insaisissables. Ce mode de décryptage est de plus doublé d'un parallèle avec le hasard par définition imprévisible, tel ce feu de l'invisible qui frappe sans prévenir et qui lui est associé. Les rapports entretenus avec le surnaturel renvoient ainsi à des savoirs nés du besoin d'agir sur un mécanisme singulier de la nature, auquel celui-ci aurait recours sous son extension magique; sur une «force impersonnelle et indéchiffrable » pour reprendre une formule de M. Mauss ${ }^{28}$ - ce qui nous rapproche de la proposition de P. F. Verger, l'hypothèse complémentaire d'un éventuel volet religieux en moins. Notre entreprise consistera à exposer les diverses implications de cette perspective.

\section{L'ENQUÊTE DE TERRAIN}

Avant d'entrer dans le vif du sujet, des précisions sont nécessaires. Elles portent sur la présence de données issues de la période coloniale, voire antérieures, à côté de celles recueillies lors des séjours sur le terrain. On pourrait naturellement se demander si les représentations collectives contemporaines s'inscrivent bien dans une continuité. Et que désignerait cette continuité ? Défions-nous cependant d'une réponse qui ferait la part belle aux discours. Peutêtre vaut-il mieux saisir pourquoi des pratiques, reflets d'idées et de valeurs fondamentales, perdurent ou tombent dans l'oubli. Une relation synthétique des circonstances de l'enquête va tenter d'éclaircir ce point.

28. Marcel Mauss, Sociologie et anthropologie, Paris, PUF, 10-53, 1950. 
Une crise politique aiguë à l'origine d'une hausse de la violence et d'une récession de l'économie (aggravée par la dévaluation de la monnaie le 11 janvier 1994) caractérise le Togo des années 1990. L'épreuve de force engagée depuis le début de la décennie entre le Général-Président Eyadéma et une écrasante majorité de la population se traduit par le divorce du citoyen d'avec l'État ${ }^{29}$. L'attitude répressive du régime pousse des dizaines de milliers de citadins qui n'ont pas fui au Bénin ou au Ghana ${ }^{30}$ à prendre le chemin du village : à la différence de la ville touchée de plein fouet par la violence politique, la campagne jouit toujours d'un calme relatif ${ }^{31}$ et, surtout, elle sait pouvoir recourir à l'agriculture d'autosubsistance ${ }^{32}-$ seule alternative envisageable à ce moment-là pour atténuer les effets dévastateurs de l'autoritarisme sur l'économie nationale. Choisir une alternative locale équivaut à un véritable défi en pays Ewé. Mais l'importance accrue de la terre va avoir un impact inattendu. Le retour de citadins à un mode de vie rural confère un subit prestige à des habitus dits ancestraux. Beaucoup redécouvrent un système de valeurs dont la ville les avait éloignés. Lorsqu'elle est centrée sur le village, la vie sociale en épouse les règles, dont d'abord le respect dû aux anciens ; il faut composer bon gré mal gré avec les détenteurs de l'autorité, responsables, parmi d'autres prérogatives, des cultes traditionnels. À la faveur d'une situation exceptionnelle de nombreuses croyances connaissent un regain de popularité, comme celle, au demeurant centrale, d'une relation de cause à effet entre le respect d'un code éthique et une saison des pluies généreuse. Face à l'incertitude du lendemain, chacun,

29. Comi. M. Toulabor, Jeux de mots, jeux de vilains. Lexique de la dérision politique au Togo, in Jean-François Bayart, Achille Mbembe, Comi. M. Toulabor, Le politique par le bas en Afrique noire, Paris, Karthala, 1992.

30. Entre avril 1992 et août 1993, au moins 200000 personnes (en majorité des loméens) se sont exilées.

31. Philippe Gervais-Lambony, «LOME. Troubles politiques et images de la ville», in L'Afrique politique. Vue sur la démocratie à marée basse, Paris, Karthala, 119-130, 1994.

32. De 1993 à 1999, si l'on considère cette seule période, les surfaces emblavées pour le maiis et le manioc (la base de l'alimentation) ont doublé en pays éwé (Direction des enquêtes statistiques et agricoles, DESA, Lomé). 
quelles que soient ses convictions religieuses, espère un don du Ciel. Et puisqu'on réside sur les terres des aïeux, il est particulièrement mal venu d'offusquer le vodu initiateur des ondées fécondantes. Sait-on jamais, la Providence pourrait bien prendre un visage local.

À l'inverse des pratiques, directement exposées aux jugements, "l'idéo-logique ${ }^{33}$ (la logique des représentations) semble offrir une meilleure résistance. Car si au nom de sa mission civilisatrice l'administration coloniale légiféra au détriment du droit coutumier et impulsa une agriculture de rente en sa faveur ${ }^{34}$, elle ne réussit par contre jamais à saper les bases de la culture. Quant aux missionnaires, à l'œuvre sur la côte dès le milieu du XVIII ${ }^{\mathrm{e}}$ siècle, leur prosélytisme connut des fortunes inégales ${ }^{35}$. Lorsqu'il n'a pas été rejeté, le christianisme a souvent pris une teinte locale, une forme syncrétique. En période de très forte instabilité le sentiment religieux se modifie de nouveau: résoudre les problèmes quotidiens prend le pas sur la quête de salut. Chacun diversifie davantage son rapport au sacré : il ne faut en aucune façon négliger les services disponibles, qu'ils proviennent d'un devin-guérisseur, d'un prêtre ou d'un pasteur - de leur côté, les Églises indépendantes ont fort bien pris la mesure de la détresse populaire en proposant un large éventail de « prestations ». Ces formes de nomadisme religieux ne sont pas nouvelles. En revanche, à la différence du passé - et ceci permet de l'affirmer,

33. M. Augé, op. cit., p. 73, 1977.

34. Arthur J. Knoll, Togo under Imperial Germany : a case study in colonial rule, Stanford Hoover Institution Press, 1978 ; Heinrich Klose, Le Togo sous drapeau allemand (1894-1897) selon Heinrich Klose (trad. fr. de Togo unter deutscher Flagge, Berlin, 1899), Haho-Karthala, Lomé, 1992 ; Ulrike Schuerkens, L'administration française au Togo et l'utilisation de la notion de coutume, Université Paris X Nanterre, «Droit et cultures », n² 23, p. 213-229, 1992 ; «La pratique "administrative-anthropologique" de la France au Togo. Les activités du gouverneur Bonnecarrère (1922-1931), Paris, Revue française d'histoire d'outre-mer, LXXX, 302, p. 55-70, 1994 ; Mahmood Mamdani, Citizen and Subject. Contemporary Africa and the legacy of late colonialism, Princeton, Princeton University Press, 1996 ; Nicoué Lodjou Gayibor (sous la dir. de), Le Togo sous domination coloniale (1884-1960), Lomé, les Presses de l’université du Bénin, p. 9-54 \& 124-136, 1997.

35. Idem, p. 71-79. 
on ne pratique plus aujourd'hui avec un état d'esprit identique à celui de la période coloniale - l'acte de foi tend à être confondu de manière systématique avec un investissement dont on exige un retour, à savoir une intervention divine pour satisfaire, notamment, des attentes auxquelles l'État demeure sourd ; une pratique courante dans les Églises indépendantes où, au Togo peut-être plus qu'ailleurs (par exemple au Bénin ${ }^{36}$ ), la course aux rites efficients confine à l'obsession. En l'espace de quelques décennies les raisons d'adhérer aux dogmes ont donc beaucoup changé : l'idée que les puissances invisibles puissent être contraintes à grands renforts de prières et d'offrandes (une pure hérésie du point de vue de l'orthodoxie, chrétienne ou ancestrale) se banalise chez les groupes sociaux défavorisés. Les surenchères (fréquemment suggérées par les ministres des divers cultes) relèvent d'une stratégie de survie dans cette société alors en attente de se libérer de l'oppression issue de ses propres rangs - une différence fondamentale d'avec la période coloniale. Il s'agit dorénavant de vivre dans un cadre où des enfants du pays tiennent les rênes du pouvoir; des individus par conséquent susceptibles, eux aussi, de recourir à des moyens identiques pour servir leurs intérêts. Au vu du jeu ininterrompu (depuis 1967) du régime tantôt sur le registre républicain tantôt sur celui de la coutume $^{37}$, toutes les supputations sont possibles.

Les maux de la société globale font l'objet d'un décodage analogue à celui en vigueur pour conférer du sens à ceux du village : la délinquance, la répression policière, les rumeurs d'enrichissement des autorités, les privilèges d'une poignée de nantis, la corruption et la fraude, les carences des systèmes éducatif et de santé, la chute vertigineuse d'un pouvoir d'achat déjà faible... sont perçues comme autant de signes d'un commerce répréhensible avec des puissances obscures - une perception du pouvoir du reste très largement

36. A. de Surgy, «La recherche de "miracles" dans les Églises chrétiennes en République du Bénin », in Religion et pratiques de puissance, Paris, L'Harmattan, p. 175-206, 1997.

37. C. M. Toulabor, Le Togo sous Eyadéma, Paris, Karthala, 1986 ; E. Adriaan B. van Rouveroy van Nieuwaal, L'État en Afrique face à la chefferie. Le cas du Togo, Leyde-Paris, ASC-Karthala, 2000. 
répandue à l'échelle du continent ${ }^{38}$. Ce mode de lecture se démarque de celui ayant prévalu au cours de la colonisation, personne ne soupçonnant alors un administrateur ou le gérant européen d'une maison de commerce de suspendre ses actes à la consultation préalable d'un féticheur ou à l'exécution de sacrifices propitiatoires ${ }^{39}$. On assiste à une actualisation (en l'occurrence la troisième ${ }^{40}$ ) de schémas pré-coloniaux ; une démarche révélatrice, par contrecoup, de l'incontestable capacité d'adaptation de la tradition, de sa plasticité. Elle n'est toutefois pleinement compréhensible que si l'on garde en mémoire les caractéristiques de la dynamique affectant désormais les rapports inter-sociétaux. Mondialisation oblige, sous la houlette de ses partenaires économiques (la France, l'UE...) et des organismes financiers internationaux (FMI, Banque mondiale...), le Togo est à son tour pris dans une spirale d'exigences peu enclines, en première analyse, à s'émouvoir du sort des démunis. À une époque où la remise en cause du moindre acquis et le mouvement pour le mouvement tendent à devenir la règle (des indices de la « modernité ${ }^{41}$ ), le repli vers le patrimoine culturel est également symptomatique à la fois du refus de l'agitation permanente et du souci de satisfaire des demandes urgentes. Pareille démarche atteste un profond désarroi. Afin de « se garantir », espère-t-on, un avenir moins sombre, tous les moyens sont a priori mobilisables, incluant de fait ceux connus depuis la période pré-coloniale.

38. J.-F. Bayart, L'État en Afrique. La politique du ventre, Paris, Fayard, 1989 ; Peter Geshiere, Sorcellerie et politique. La viande des autres, Paris, Karthala, 1995.

39. D'après les Africains, les Blancs disposaient d'une magie bien à eux. La conversion d'une partie de la population au christianisme (presque exclusivement au sud, le nord étant sous l'influence de l'islam) s'explique très souvent par le désir de bénéficier de son hypothétique force grâce à laquelle les Blancs pouvaient faire étalage de leur réussite matérielle. Cette idée perdure jusqu'à aujourd'hui, à telle enseigne que les Églises indépendantes s'appuient sur les miracles mentionnés dans la Bible et dans le Nouveau Testament pour construire leurs liturgies et revendiquer la « puissance » de leurs rites.

40. La première actualisation ayant eu lieu du temps des Allemands puis la seconde du temps des Français, chaque style de colonisation requérant une adaptation spécifique.

41. Georges Balandier, Le détour. Pouvoir et modernité, Paris, Fayard, 1985. 
L'enquête fut donc réalisée à un moment où le Togo traversait une phase particulièrement critique. Sur le plan de l'investigation, l'instabilité du contexte a permis contre toute attente de mieux saisir la visée des propos et des usages. Parce qu'ils prenaient soudain un relief singulier, ils exprimaient au plus près les schèmes de pensée d'une population condamnée à rechercher dans son héritage les clefs utiles à la compréhension d'une situation devenue chaotique. C'est la place du feu, pièce maîtresse de cet héritage, dans les schèmes de pensée que nous allons maintenant découvrir.

\section{L'ÉNERGIE MYSTÉRIEUSE}

Les Ewé scindent le feu en deux grandes catégories : les feux physiques (l'éclair, le feu de brousse, le feu de la forge, le feu domestique...), directement observables, et les feux invisibles (les «plantes de feu», les «pierres de foudre », le feu des métaux, le feu du guérisseur, le feu du sorcier et les feux des esprits), conçus comme une variante des premiers, tapis au cœur de la matière ou aux mains des puissances obscures. Immatériels, ces derniers interviennent lors de pratiques magiques, thérapeutiques ou bien à l'occasion de rituels, sans pour autant revêtir un caractère religieux ni faire l'objet de la moindre dévotion. Il n'y a d'ailleurs pas de dieu du feu stricto sensu au sein du panthéon ${ }^{42}$. Qu'il se manifeste au grand jour ou sur un mode invisible, le feu est toujours pensé comme le principe vital responsable de la dynamique de l'univers ${ }^{43}$. Consécutif au geste créatif de Mawu (Dieu), il renvoie à la conception philosophique du feu principiel, au chaos organisateur lors des premiers instants du monde. Car bien que mystérieux et désormais symbole du désordre en général ${ }^{44}$, cet élément aurait pourtant eu

42. Comme il n'y a pas de dieux de l'air, de l'eau et de la terre, même si de grands vodu ont le pouvoir de les manipuler; ce qui ne signifie donc pas que la pluie, le vent... soient systématiquement leurs faits.

43. Insistons sur le caractère profane du feu. Pas même le métallurgiste (forgeron ou bijoutier) n'y voit une émanation du sacré, malgré la forte charge d'ésotérisme qui connote pourtant ses activités.

44. C. Rivière, op. cit., p. 175-190. 
initialement un impact positif. On lui prête d'avoir eu un pouvoir génésique qui se serait illustré dans la continuité de la foudre produite par le contact de la terre et du ciel ${ }^{45}$. Toujours d'après les contes populaires, ce choc terrible donna l'impulsion initiale à toute transformation cosmique. Considéré comme le prolongement de la puissance de la foudre, il participe avec la terre, l'air et l'eau aux interférences fondatrices de l'ensemble organique constitutif du monde. D'aucuns affirment voir aussi en lui le dénominateur commun aux trois états de la matière ${ }^{46}$, conférant de la sorte à cette philosophia perennis le caractère d'un matérialisme organique comparable, sous certains aspects, à celui de la Chine taoïste ${ }^{47}$ ou encore à celui imaginé par Héraclite d'Éphèse (la dimension tragique en moins).

Les Ewé élaborent un discours faisant ressortir moins le désir de connaître qu'un besoin de sens, «le besoin de s'y reconnaître » pour reprendre une formule très générale de $\mathrm{M}$. Augé ${ }^{48}$ : un phénomène physique inaugural, suivi de réactions énergétiques (en l'occurrence thermodynamiques), a joué un rôle-clef dans la genèse du monde et

45. Lors de son enquête chez les Fon, B. Maupoil (op. cit., p. 72) relève la même idée, sous une forme allusive, dans le cas d'un mari trompé projetant de se venger : "Nu de de nu so mo a! Fi e ji nukù sù, kpodo ayikùgbà kpa, yi kpele dele, a tù nu e no ti do le a? » («Ce n'est pas une affaire! Sais-tu ce qui se passe là-bas, quand le Ciel et la Terre se rencontrent ?»). Nous le verrons à propos de la Loi, la foudre que ne manquerait pas de recevoir l'assassin est celle du vodu Héviesso (Xevioso en pays fon, Shango en pays yoruba), qui mobilise le feu originel, ici confondu avec son propre feu, à des fins de justice. Il faut le souligner, l'existence du feu ne relève d'aucune volonté divine mais elle est, là encore, si l'on peut dire, le fruit d'un accident du cosmos.

46. Les Fon mentionnent à l'appui de cette thèse le vodu Gbaadu (ou Odu), d'origine yoruba, symbole de l'union féconde du ciel et de la terre. À son sujet, B. Maupoil (op. cit., p. 90) écrit : «Il est en rapport avec un culte déchu des quatre éléments et des quatre points cardinaux. » Il ne put cependant obtenir davantage de précisions pour étayer sa thèse, les connaissances de ses informateurs demeurant lacunaires et contradictoires. Le plus érudit d'entre eux lui livra la définition suivante : "Gbaadu est formé d'Eau, d'Air, de Terre et de Feu. »

47. Joseph Needham, La science chinoise et l'Occident, Paris, Seuil, p. 13-52 \& 223-261, 1969.

48. M. Augé, Le sens des autres. Actualité de l'anthropologie, Paris, Fayard, p. 131, 1994. 
il lui imprime depuis lors son rythme. Notre objectif ne consiste pas à discuter la thèse d'une connaissance inconsciente des lois scientifiques par les « primitifs ${ }^{49}$, ni celle d'une « science du concret ${ }^{50}$ ou encore celle de "l'humain comme ordonnateur paradigmatique ${ }^{51}$, il consiste à saisir des pratiques et des discours à partir d'un point fondamental : pour les Ewé l'élément feu est ambigu (« ni bon ni mauvais ») quant à son essence et ambivalent («bon et mauvais ») quant à ses effets. Nombre de locutions retiennent son caractère menaçant pour signifier le danger, la destruction, la douleur, l'inquiétude et le mal : dzoto ou dzôtoé (le « volcan »), dzo fé dódo (l' « éruption »), dzoxoxó akpa ou dzototo akpa (la « surchauffe »), dzoxí (l' «étincelle »), dzobịbi ou dzofadé (la « flamme »), dzo gà ou dzodzèàfé ou encore dzotogbé (l' « incendie »), dzokà (la « braise »), dzotóto (le «brûlage »), dzobị (la «brûlure»), dzodélàmè (l'« échauffement»), dzoxoxo (le «brasier », l' « inflammation »), $d z o$ le...gbo (« trembloter »), dzo dé dzi («sursauter »), domèdzoè (la « colère »), dzoma vô (l' « enfer »)... À l'opposé d'autres traduisent ses bienfaits : dzokeklé (la «lumière », la « lueur»), dzodódo (le « chauffage »), dzo (kplé) (« chaleureusement»), dzoko («assouplir »), dzokodzoko (l' « assouplissement »), dzodofé (la « cuisine »)... Les multiples combinaisons autour du mot $d z o$ expriment l'impossible définition de ce qu'il désigne réellement. Leur impuissance laisse pleine et entière sa place au mystère.

L'ambiguïté autour du feu renvoie à la difficulté de le connaître comme tel, une difficulté à laquelle se heurte au demeurant quiconque essaie de déchiffrer un phénomène physique. L'homme reste inévitablement tributaire de ses expériences. Ce qu'il observe n'est pas la nature en soi mais seulement la nature exposée à ses méthodes d'investigation : d'où ici comme ailleurs des « relations

49. Lucien Lévy-Bruhl, La mentalité primitive, Paris, Alcan, 1938 ; B. Malinowski, Magic, Science and Religion, New York, Doubledday \& Co, 1955.

50. Claude Lévi-Strauss, La pensée sauvage, Paris, Plon, 1962.

51. Robin Horton, African Traditional Thought and Western Science, Africa, 37 (1-2), 1967, p. 50-71 \& 155-187 ; Tradition and Modernity Revisited, Cambridge (Mass), MIT Press, 1982, p. 201-260. 
d'incertitude $»^{52}$ et l'impossibilité de concevoir autre chose qu'un modèle approximatif ${ }^{53}$, a fortiori là où « les poèmes cachent les théorèmes ${ }^{54}$. D'où l'ambivalence. Si l'on apprécie le feu qui cuit et qui réchauffe, à l'inverse on craint celui qui dévaste ou, par exemple, celui du sorcier qui entraîne la mort : en quelque sorte, le feu devient ce que les hommes en font s'ils le manipulent. Son caractère a priori dangereux est renforcé du fait que sa maitrise parfaite relève de l'utopie. Dans le meilleur des cas il est envisageable de le contenir ; un exploit que seul le forgeron peut accomplir. Le terme dzogbèdé, également utilisé pour désigner le métallurgiste, l'atteste : dzogbèdé, littéralement «feu » $(d z o)$ et «forgeron » (gbèdé), afin de traduire que l'artisan forge moins la matière (en l'occurrence le fer) que le feu qu'elle abrite. À travers le prisme de cette source d'énergie et les conséquences de ses usages, le monde (y compris le vivant dont l'homme) apparaît donc soumis à une oscillation permanente entre un état stable et un état instable. En d'autres termes, ni la nature ni la société ne connaissent un équilibre définitif. Ils sont pris dans un mouvement que les Ewé comparent à une respiration ( $g b \hat{g} g b \hat{o})$, à un souffle semblable à celui qui trahirait la présence des puissances invisibles.

L'importance extrême du feu ne doit toutefois pas nous égarer quant à sa place au sein du système religieux. Répétons-le, il n'y a pas de dieu du feu en dépit du fait qu'on associe la foudre au vodu Héviesso. Au demeurant aucun autre élément (l'air, l'eau et la terre) n'est déifié, pas même sous une forme allusive ou résiduelle. S'il existe encore des vestiges de croyances en rapport direct avec les forces de la nature - la remarque vaut également pour les cultes introduits au Nouveau Monde ${ }^{55}$, ils se situent peut-être au niveau de l'idée d'une possible intervention du numineux sur des phénomènes

52. Werner Heisenberg, Physique et philosophie, (trad. fr.), Paris, Albin Michel, p. 50, 1961.

53. Isabelle Stengers, L'invention des sciences modernes, Paris, Flammarion, p. 183-189, 1993.

54. Gaston Bachelard, La psychanalyse du feu, Paris, Gallimard, p. 12, 1949.

55. Alfred Métraux, «Le culte Vodu en Haïti », La Revue de Paris, août 1953 ; P. F. Verger, op. cit., 1957. 
naturels dévastateurs (la tempête, l'ouragan...). Soyons précis sur le sens des sollicitations de l'invisible: on n'attend pas des vodu qu'ils interviennent au motif qu'ils seraient les animateurs habituels des éléments, on sollicite leur action parce qu'ils détiendraient le pouvoir de les apaiser. L'ensemble de ces précisions suffit-il alors pour avancer que les forces de la nature composèrent la base de l'édifice religieux ? Pour reprendre une interrogation de P. F. Verger, firent-elles jadis l'objet de croyances particulières? Il y a là, me semble-t-il, un pas à ne pas franchir en l'état actuel de nos connaissances. La prudence s'impose a fortiori puisque le feu occupe le devant de la scène d'une manière très spécifique. Un moyen de mieux saisir ce qu'il représente consiste à observer un certain nombre de pratiques. Cela suppose l'adoption préalable d'outils d'analyse appropriés, car elles demeurent incompréhensibles si l'on s'attache aux catégories de «bien », de «mal », de "faute originelle », de « divine providence »... Il est préférable de leur substituer celles d' " efficacité », de "force », de «lutte » pour l'existence, etc., étant admis que la nature peut être sinon domestiquée du moins apprivoisée - «assagie » écrit P. F. Verger ${ }^{56}$ - grâce à des associations d'éléments de prime abord incompatibles pour un esprit occidental. L'étude d'exemples du jeu sur le «chaud» et le « froid » auquel les Ewé recourent en maintes circonstances illustrera cette approche.

On retiendra dès à présent deux résultats : pour les Ewé l'univers est le lieu où se manifestent des phénomènes énergétiques affectant les êtres et les choses, d'une part, et le feu déterminant la source de ces phénomènes, les humains ne subissent en aucun cas l'emprise d'un champ de forces initié par une multitude de divinités, d'autre part. Chaque individu doit bien plutôt composer avec des flux d'énergie sur lesquels, du reste, il reconnaît des limites à sa capacité d'intervenir. Cette conception tranche avec celle d'ordinaire admise d'une société somme toute condamnée à satisfaire les volontés, voire les caprices, du surnaturel. Au contraire, il faudra y revenir, cette approche expressément ouverte, dynamique, souligne

56. Op. cit., p. 12, 1957. 
l'inévitable implication de l'individu. Et c'est au cours de l'exposé des relations entretenues avec différents esprits que nous la relèverons.

\section{LA SOURCE D'UN DYNAMISME UNIVERSEL}

La tradition distingue deux étapes dans la création du monde : la première est du ressort de Mawu qui conçoit le ciel et la terre - et, on le suppose, le règne végétal et le règne animal dans la foulée - et la deuxième résulte d'une expression du hasard née de leur rencontre. Seule la seconde est fertile, propre à la vie, étant admis que le feu issu de la foudre aurait induit un perpétuel mouvement ${ }^{57}$. Apparait donc une cause initiale sous la forme d'une source d'énergie. Sur ce dernier point, avec les réserves déjà émises, A. de Surgy se rapproche de la tradition lorsqu'il déclare : «En l'occurrence, il convient, à mon avis, de ne faire appel à la notion d'énergie que pour désigner la sorte de bouillonnement générateur communicatif qui agite en permanence la nature. ${ }^{58}$ » Reste que ce « bouillonnement générateur communicatif » n'a rien de « spirituel » au dire des Ewé.

Puisqu'il est le prolongement de la foudre à l'origine, le feu appartient à la sphère céleste ; un espace animé par un dynamisme incompréhensible, pense-t-on, des mortels. À l'instar de fréquents discours en Afrique ${ }^{59}$, les Ewé identifient eux aussi le ciel à un lieu en première lecture équilibré, de façon continue ni nuageux ni serein, ni chaud ni froid : les éléments qui le composent (le vent, les nuages, la pluie, l'orage, l'arc-en-ciel et les astres) et l'alternance du jour et de la nuit qui modifient sa couleur (le blanc et le bleu signes de la pureté, le noir en relation avec la mort et les esprits) en donnent l'image d'un espace globalement stable. Mais ce lieu où dominent d'ordinaire l'ordre et la régularité connaît parfois des soubresauts : la bourrasque, la foudre, le tonnerre... Ces désordres

57. Ce schéma diffère sensiblement de celui d'ordinaire rencontré en Afrique. Très souvent, le Dieu créateur se retire au ciel et délègue la direction de sa création - son cours - à des puissances subalternes lui demeurant subordonnées. Rien de tel ici où nulle entité ne prend en charge la direction de tel ou tel secteur de l'univers.

58. Op. cit., p. 92, 1995.

59. Dominique Zahan, Le feu en Afrique, Paris, L'Harmattan, p. 25-41, 1995. 
masquent l'ordre, ils le perturbent, provoquant ainsi une succession de chaos, de retours comparables au tumulte originel. Néanmoins, à chaque fois, le monde resurgit régénéré et ordonné à nouveau ; il retrouve son point d'équilibre thermique synonyme de paix. Le feu détermine le ressort de cette dynamique en tant que source des manifestations intempestives. On retrouve cette caractéristique dans les noms attribués aux mois de l'année correspondant au plus fort de la saison sèche, quand les assauts du soleil jaunissent la végétation : dzomè (décembre, «dans le feu »), dzovè (janvier, «le second feu»)

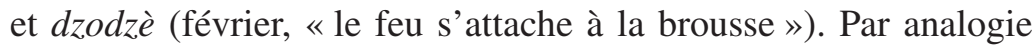
avec le ciel, une année constitue un cycle stable avec ses périodes de chaleur et ses périodes de fraîcheur.

Dans le droit fil de cette logique, le feu rythme également le cycle des saisons (la saison sèche et la saison des pluies) conçu sur le mode d'une alternance entre chaud et frais, sec et humide. Ce symbole de la puissance génésique, le ciel l'abrite sous forme première d'énergie pure de la même façon qu'il abrite l'horizon, l'éclair, le tonnerre, les nuages, la pluie ou encore les astres. Bien davantage qu'un grand réservoir des eaux, il est le réceptacle du principe cosmique, du chaud et du froid matérialisés par le soleil et la lune (respectivement sexualisés en un ciel mâle et chaud et un ciel femelle et frais) et aux apparitions rythmées par la succession des jours et des nuits. De la sorte, l'espace offert aux hommes par Mawu bénéficie de l'influence du feu céleste: il l'irradie. L'implication du feu dans toute forme de vie réapparaît dans la langue : àgbè signifie indifféremment la vie, l'existence et l'énergie et dé $d z o$ (dé, «mettre », et $d z o$, «feu ») animer, activer au sens large du terme. De ce point de vue, le feu donne vie à la matière ; le mouvement lui est consubstantiel comme l'indiquent les mots dzodzo (« départ»), dzodénùmèla ( « animateur »)... ou encore dzodzro pour le vœu, l'envie, le désir, indispensables moteurs de n'importe quelle entreprise ; Dzo nye nù̀sé ${ }^{60}$ («le feu

60. Le mot nùsé (littéralement « corps-force ») permet d'exprimer l'existence de la «force» qui se déploie à partir de l'élément physique «feu » ( « corps »). En préférant nùsé à àgbè (« énergie », « vie »), le locuteur veut ici mettre l'accent sur une relation de cause à effet. 
c'est à la fois une énergie et une force »). Il n'y a pas là de séparation ontologique entre le milieu et l'individu : le second se fond dans le premier et inversement, l'un et l'autre participant à l'existence d'un tout indissociable. Ceci rejoint la proposition très générale de G. Balandier selon qui il y aurait « une analogie entre l'ordre devant prévaloir dans la société et celui de la nature, donnant ainsi à croire qu'il existerait une nature sociale à laquelle on ne commande qu'en lui obéissant ${ }^{61}$. L'individu étant pris dans des flux d'énergie, à son modeste niveau il doit composer avec les pulsations d'un milieu face auquel il a conscience de ses faiblesses.

L'idée du feu source du dynamisme universel ne se limite pas au seul monde physique. Les Ewé conçoivent que les puissances invisibles puissent également y être soumises et à leur tour en faire usage. Sur ce point A. de Surgy propose un schéma sensiblement identique : "Tout autant qu'aux organismes vivants, [l'énergie spirituelle] est indispensable aux agents désincarnés qui veulent encore se mêler du devenir du monde. ${ }^{62}$ » Et d'ajouter :

«Grâce à l'énergie [que les agents désincarnés] ont eu la force d'amasser, ils parviennent à modifier non seulement l'engendrement au hasard des événements mais encore l'activité de ceux qui les gênent ou tentent de leur nuire..$^{63}$ » « En effet, la puissance d'interaction qui est infusée à la nature [...] revêt alors l'aspect d'un pneuma qui s'exhale des choses et les enveloppe d'une sorte d'aura propre à attirer sur elles l'attention des êtres. ${ }^{64}$ »

Nous avons déjà dit quelques mots sur la notion d' «énergie spirituelle ». Mais au-delà des divergences, on s'accorde à admettre que l'invisible et le visible ont en commun de puiser leur moyen d'expression dans un facteur énergétique. Sous un autre angle et chez les Fon, B. Maupoil avait abouti à un constat similaire sans 1988.

61. G. Balandier, Le désordre. Éloge du mouvement, Paris, Fayard, p. 119,

62. Op. cit., p. 91, 1995.

63. Idem, p. 92.

64. Ibid, p. 96. 
toutefois en tirer une conclusion sur le rôle du feu qu'il appelait la « flamme divine $» 65$.

C'est au feu et plus particulièrement à son prolongement à travers la chaleur que les Ewé attribuent au monde son existence, assimilant de la sorte la matière à une « matière-puissance ». C'est également au moyen du feu que les puissances invisibles se manifesteraient. Insistons sur ce point, rien n'émanant d'une quelconque volonté divine ne sous-tend ce dynamisme. Toute chose s'y trouve inévitablement soumise par définition. Grâce à lui, la matière prend vie avec toutefois le risque omniprésent qu'un trop plein de feu n'entraîne la destruction. Sous une forme symbolique, la perpétuation de la vie repose par conséquent sur la quête ininterrompue d'un équilibre toujours précaire entre un excès et un déficit de chaleur annonciateurs l'un et l'autre de la mort. Cette conception d'un monde sans cesse menacé de courir dans un sens ou dans un autre vers sa perte, on la retrouve au niveau des caractéristiques prêtées à l'espace sublunaire.

65. Écoutons B. Maupoil (op. cit., p. 61) : «Le phénomène de la possession nous amena à poser la question suivante: "Jusqu'à quel point le vodu vient-il en vous ? Jusqu'à quel point votre propre désir ne le crée-t-il pas ?"

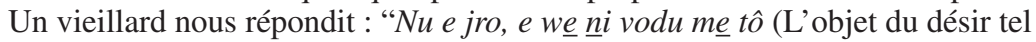
est le vodu de chacun)". Et encore : "Vodu towe lo ayi towe me we de. Gbe nô dekpa me $a$; ayi me we nô doxo do (Ton vodu est dans ton rein. La Vie ne chuchote pas à l'oreille des gens : c'est dans ton rein même qu'elle parle)". » Cette dernière phrase étant un proverbe, afin de s'assurer de son sens B. Maupoil proposa l'interprétation suivante qui fut admise par son informateur : «Ce n'est pas au-dessus de nous, c'est au fond de notre cœur que brûle éternellement la flamme divine. » Ce que B. Maupoil désigne par l'expression « flamme divine » ressemble à s'y méprendre au feu chez les Ewé. «Divine » exprime plutôt ce que le feu peut avoir à la fois d'incompréhensible, d'insaisissable et d'omniprésent en toute chose, autant de propriétés qui le rapprochent effectivement du divin sans toutefois lui en conférer la qualité. On comprend dès lors que l'interlocuteur de B. Maupoil ait acquiescé. Au demeurant, pour les Fon aussi le feu est l'agent de la dynamique qui affecte le monde matériel et le monde immatériel. 


\section{ZONES CHAUDES, ZONES FRAÎCHES}

Sous l'effet des influences cosmiques, le monde sublunaire abriterait des parcelles de feu dans des proportions variables. Les Ewé répartissent ainsi la terre en secteurs diversement chauds. Par endroits, le sous-sol recèle des zones chaudes dues à la présence de fer ou de pierres de foudre. Supposés venir du ciel à l'occasion des orages, ces minéraux seraient naturellement chargés de feu céleste : le fer est «matière-puissance »- principal motif des précautions prises lors de son emploi - et les pierres de foudre font, pour ce motif, l'objet d'un culte par les prêtres du vodu Héviesso. En surface, il y a d'un côté le village et les champs, de l'autre, la brousse ou la forêt. Gagné au préalable sur la végétation, le village est un lieu clos où chaque concession familiale constitue a priori un espace à l'abri des feux des puissances errantes. En revanche à l'extérieur des zones habitées règnent diverses entités. Le contact avec leurs feux présente toujours un risque pour le non-initié : les termitières accueillent les Aziza qui désignent au guérisseur les plantes médicinales et, aux carrefours, aux points d'eau... résident les $\mathrm{Na}$, dont la mobilisation par le sorcier permet aux forces dangereuses d'intervenir dans la société des hommes. Entreprendre la culture d'un lopin de terre signifie clairement franchir les limites d'un espace socialisé et, de fait, s'exposer aux dangers d'un univers dont la marche échappe à la volonté humaine. Y poussent deux catégories d'essences classifiées en fonction de leurs propriétés et, par suite, en fonction de leurs emplois possibles; un procédé commun aux divers groupes de la région et qui se démarque de celui élaboré par Linné $^{66}$. On distingue celles dites «fraîches » (àmà fàfà), au contact doux, manifestant des propriétés sédatives ou rééquilibrantes (par exemple les plantes aquatiques), synonymes de paix et de bienêtre, sources de la fortune, du bonheur, de la gloire, de la chance... de celles dites « chaudes » (àmà dzodzo), ayant des effets urticants ou enfiévrants (par exemple les épineux), synonymes de conflits, de maladies, de la misère, du bavardage indiscret... Les Ewé ont par

66. P. F. Verger, Ewé : le verbe et le pouvoir des plantes chez les Yoruba, Paris, Maisonneuve et Larose, p. 26-28, 1997. 
conséquent une vision ambivalente des espaces vierges : à la fois accueillants et hostiles, source de vie et de mort, lieu de l'ordre et du désordre. S'y aventurer nécessite de solides protections afin de pouvoir affronter l'inconnu - les chasseurs en fournissent le plus bel exemple, leurs succès tenant au moins autant à une capacité à braver les dangers qu'à une connaissance de la faune.

Le milieu présente cependant une relative stabilité : l'emprise de la puissance du feu (matérialisée ici par le soleil) auquel il doit son existence voit ses effets atténués (avant qu'ils ne deviennent dévastateurs) grâce à l'action apaisante des pluies et au cycle diurne-nocturne. Cette lecture du règne végétal aboutit à une modélisation servant de canevas pour conceptualiser les travaux agricoles. Mettre en culture une terre signifie accaparer un univers où s'entrecroisent harmonieusement le principe chaud et le principe froid ; ce qui implique d'abord de détruire son équilibre avant de le recomposer sur un mode artificiel. Il faut bien distinguer cette activité de la cueillette : l'acte de cueillir se limite à induire une perturbation, ce qui suppose toutefois le respect d'un minimum de règles à cause des suites envisageables. À telle enseigne que l'herboriste, « en état de pureté » (c'est-à-dire s'étant abstenu de relations sexuelles la nuit précédente), prend soin de déposer de petites offrandes là où il prélève des plantes - une précaution également prise lors de la fondation d'une unité d'habitation, d'un village ou lors de la mise en culture d'un site - afin de ne pas déclencher le feu des puissances de la brousse et, de la sorte, outre le risque de subir l'attaque d'un feu magique, de ne pas provoquer la stérilité du sol. Cueillir et cultiver déterminent donc deux rapports distincts avec le milieu. Ils renvoient à ce qui distingue le «champ » (àgblè) de la «brousse » (gbè); de ce lieu auquel est attribué un feu particulier, dzogbè (le «feu », dzo, de la «brousse », gbè), pour exprimer la présence des puissances du désordre. Cette distinction s'illustre très précisément dans le cycle de rotation entre l'un et l'autre : le champ relève du domaine de la culture le temps d'une saison agricole puis il est rendu à la nature, la brousse, jusqu'à l'année suivante. Un débroussaillement préalable ou, éventuellement, un feu - toujours après un petit rituel pour se 
concilier les esprits - constitueront le prélude à son retour dans le pôle culture.

Le froid et le chaud renvoient respectivement à l'ordre indispensable à la société et au désordre susceptible de l'affecter. Sous forme minérale ou organique, la nature recèlerait déjà en son sein ces contraires, symbolisés par la fraîcheur associée à la cité organisée et par la chaleur associée, elle, à l'anarchie. Cette analyse resurgit à propos de la cueillette; elle repose sur l'idée que, l'intervention humaine perturbant un ordre supérieur incarné dans la nature, il faut, de quelque manière, rétablir l'équilibre initial. La démarche de don-contre-don destinée à apaiser les puissances locales traduit explicitement une précaution majeure : éviter de générer un désordre aux conséquences désastreuses. Ce souci de ne rien entreprendre qui puisse induire une rupture transparaît à un degré égal dans le cadre des activités agricoles.

\section{LA NATURE RECOMPOSÉE}

Après avoir exposé la place du feu dans la conception Ewé de l'espace physique, observons maintenant la place du chaud et du froid lorsque l'homme procède à une recomposition de la nature telle qu'elle se présente avec l'agriculture. Ce type d'activité l'illustre de façon exemplaire: transformer le milieu ne saurait se limiter à obtenir le principal moyen de subsistance. Par-delà l'obtention de récoltes, le cultivateur engage un autre processus ; il importe certes de reconnaître l'appartenance de l'homme à l'univers mais, tout autant, de socialiser l'homme à travers ce dernier.

La culture de telle ou telle plante signifie se placer dans une perspective soit «chaude » soit «fraîche », selon que le contact avec la plante ait un effet apaisant ou qu'il déclenche une irritation. La physionomie du jardin d'ignames en fournit l'illustration la plus probante : entre les buttes où poussent les essences « froides » (les tubercules), on relève la présence d'essences «chaudes » comme par exemple le piment. Leur répartition traduit une modélisation du social. L'igname renvoie aux règles à observer afin que le vivre- 
ensemble soit envisageable : tolérance, honnêteté, respect de l'autorité, des usages... Aliment noble, il figure la continuité du lignage ainsi que la permanence des liens entre les vivants et les morts. Son bulbe est symbole de verticalité, de santé et de pérennité. On assimile la communauté villageoise à sa tige et son tuteur au chef. La consommation des premières ignames est ritualisée ; l'offrande partagée entre les esprits et les officiants exprime sur un mode solennel le souci de voir le groupe vivre en paix : «Que leur consommation ne donne pas de maux de ventre. ${ }^{67} \gg$ Ce type de mal étant en général associé à l'action d'un feu consécutive à une attaque sorcière. À l'inverse, il ne viendrait à l'idée de personne d'offrir les premiers piments de la saison! Car le piment rappelle les menaces pesant sur le lien social : le vol, le meurtre, la zizanie, la sorcellerie, etc., rapportés à des débordements de feu. Avant de voir des déterminations techniques et écologiques indissociables des représentations sociales et religieuses ${ }^{68}$, il conviendrait plutôt de souligner la présence d'éléments froids assimilés à l'ordre et celle d'éléments chauds assimilés au désordre. Non seulement observe-t-on une copie de la nature sous l'angle d'un espace clos et globalement équilibré mais, cette recomposition entend aussi traduire le rapport entre les comportements indispensables au maintien de la cohésion du groupe et ceux toujours susceptibles de la dissoudre.

À une société de végétaux correspond une société humaine. La répartition sexuelle des tâches utiles à la préparation du terrain puis celle relative au soin des plants reflètent la teneur des relations hommes-femmes sur lesquelles le groupe s'appuie : aux hommes les gros travaux, aux femmes le sarclage. Il en va de même pour l'attribution des essences, le champ d'ignames l'illustrant une fois encore sans équivoque : aux hommes la culture du tubercule, aux femmes celle du piment ; étant entendu que les hommes, à l'inverse des femmes, seraient moins prédisposés à entretenir des relations avec les puissances occultes - un point sur lequel il conviendra de

67. J. Spieth, op. cit., p. 36, 1906.

68. Buno Martinelli, Pour une anthropologie de la pluralité technique. Le cas de la culture de l'igname au sud-Togo, Université catholique de Louvain, Cultures et développement, XIII (4), p. 633-659, 1981. 
se pencher. Par le biais d'un mode d'appropriation de la terre, on exprime simultanément et les tensions et les lois inhérentes à toute vie communautaire. À l'aide de techniques culturales, le groupe transpose également la vision qu'il a de lui-même : les rapports interpersonnels sont pris dans la dynamique de l'univers. Cela n'a rien de très étonnant si l'on note qu'il n'y a pas ici de dualisme chairesprit et que l'individu admet être l'une des multiples composantes du milieu. L'agriculture revêt une importance capitale manifestement irréductible à un simple moyen de subsistance : chacun visualise de manière concrète ce sur quoi repose la pérennité du groupe (l'observation d'attitudes en faveur de l'ordre incarné dans l'igname), d'une part, et chacun est de facto renvoyé à une lecture globale du monde en termes d'une oscillation permanente entre le chaud et le froid, d'autre part. L'objectif poursuivi est sans rapport avec un quelconque souci de réaliser un usage optimal des terrains - au demeurant le problème de leur disponibilité ne s'est jamais posé, du moins à la période pré-coloniale. Les techniques culturales ainsi que la symbolique attachée aux essences réfléchissent un mode d'être et de penser ; des pratiques du reste largement répandues audelà de la sphère Ewé et même africaine ${ }^{69}$. Par ailleurs, on relèvera le caractère profane de cette approche. S'il existe bien un rapport aux puissances surnaturelles avec lesquelles serait conclu un pacte afin d'exploiter le sol, il n'en demeure pas moins que le sens de ce rapport dérive d'une lecture du monde en termes de flux d'énergies auxquels, rappelons-le, on les dit également soumises.

\section{UNE DONNÉE INCONTOURNABLE}

Appréhender la nature sous l'angle d'une discursivité des esprits serait visiblement erroné. Sans pour autant verser dans un déterminisme par définition réducteur, force est néanmoins de reconnaître

69. André-Georges Haudricourt, «Domestication des animaux, culture des plantes et traitement d'autrui », L'Homme, II (1), p. 40-50, 1962 ; « Nature et culture dans la civilisation de l'igname : l'origine des clones et des clans », L'Homme, (4), p. 92-104, 1964. 
que des considérations d'ordre physique assument un rôle central. L'approche en quelque sorte thermodynamique assure d'autant mieux une médiation des rapports entre le visible et l'invisible que, les deux sphères étant par hypothèse soumises au même dynamisme, une relation de continuité et donc de sens peut alors être établie de l'une à l'autre. Conséquence dès lors attendue, la réciprocité est non seulement envisageable mais surtout inévitable: l'invisible peut intervenir sur le visible et inversement. Toutefois, le second est en permanence placé sous l'étroite surveillance du premier ; un atout que celui-ci détient au seul motif que son feu étant omniscient, il peut à n'importe quel moment se manifester pour signaler un désordre. Cette omniprésence du feu, chacun y est en effet confronté jusque dans ses relations avec autrui.

Les préoccupations d'ordre social passent par la recherche constante d'un équilibre énergétique idéal. La place accordée à tout ce qui touche de près ou de loin à la vie l'illustre de manière significative. Dans son rapport aux plantes l'individu joue d'abord sur des échanges thermiques. La relation à l'invisible vient comme en surimpression : c'est parce que tel ou tel esprit est susceptible d'activer son feu qu'il faut, soit ne rien faire qui lui fournisse un prétexte pour attaquer, soit tenter d'en contrecarrer l'action nuisible. La phytothérapie en témoigne. Les Ewé traitent en effet l'essentiel des pathologies à l'aide de plantes ${ }^{70}$, auxquelles sont très souvent associés, P. F. Verger l'a également observé chez les Yoruba ${ }^{71}$, des travaux maléfiques ou bénéfiques tendant à estomper la frontière entre médecine et magie. De la pathologie la plus banale à celle mise sur le compte d'une entreprise sorcière ou de l'ingérence d'un vodu, le diagnostic porte toujours in fine sur la manifestation d'un déséquilibre énergétique. De manière générale souffre d'un mal celui dont le corps subit un excès de chaleur, réel ou magique. En pareil cas, la posologie habituelle consiste à consommer des plantes froides sous différentes formes (aliments, tisanes, onguents...).

70. Bernard Roussel, «Des dieux à l'homme. Les plantes du vaudou », Revue du CCVS, (7-8), p. 47-49, 1993-1994.

71. Op. cit., p. 63-79, 1997. 
Si le traitement échoue, il faut alors reconsidérer l'origine des troubles. Et la cause avancée renvoie dans ce cas à l'hypothèse d'une attaque par un feu magique. On pense à l'action d'un feu affectant le «principe vital» (klà) et fragilisant l' « âme» (luvho ) par un déséquilibre entre l' « esprit de vie » (agbé luvho) et l' « esprit de mort » (kù luvho), au profit de ce dernier. Sous peine de voir la mort l'emporter, il convient soit de s'allier les bonnes grâces du vodu impliqué - s'il a été négligé ou si une transgression a été commise, soit de repousser le mauvais sort. Le processus curatif suit un protocole immuable : après avoir déterminé l'origine de l'attaque - une opération dévolue à un « géomancien » (Afà bokono), on procède selon deux directions : l'une cherche à éteindre le feu magique dans le corps du patient et l'autre à apaiser l'esprit dans le but de l'éloigner. Avant de procéder à des ablutions à base de plantes froides pour «rafraîchir le corps », le «guérisseur» (dôdàla) procède à des aspersions de décoctions de plantes chaudes - préalablement déchiquetées (parfois nouées), dans le souci d'affirmer la victoire sur le désordre, afin d'entrer en communication avec l'entité invisible. Au cours de la cure (elle peut s'étaler sur plusieurs jours, parfois quelques semaines), des sacrifices ne sont pas rares si la puissance impliquée est un vodu. En effet, malgré tout, il importe de maintenir opérationnel son principe actif, son «feu » $(\text { dzositro })^{72}$, en lui offrant ce qui le maintient en vie : on le nourrit notamment de «sang » (vù) pour qu'il dispose de l'aptitude à mobiliser son feu. Le retour à la normale traduit la prise en compte d'un avertissement visiblement conceptualisé : par le désordre que lui-même provoque en activant son feu, le vodu prévient soit l'individu d'une négligence à son endroit, soit la communauté de l'apparition d'une menace contre son intégrité à travers le mal qui affecte l'un de ses membres. À l'exception du rituel réparateur en faveur d'un vodu suite à une faute commise par action ou par omission envers lui, ou en faveur du groupe suite à la transgression d'une règle, la lutte contre l'effet d'un « feu nuisible » (dzo vô) s'inscrit dans les pratiques

72. A. de Surgy (op. cit., p. 77, 1995) parle d'un « corps spirituel» ou « noétique » (de noêtikos, « doué d'intelligence ») qui serait le véritable agent de l'expression du surnaturel. 
visant à se défendre contre la sorcellerie. Ici le feu magique est identifié à une volonté de nuire, à une source de désordre d'un autre type. On soupçonne l'intervention d'un «propriétaire d'un feu» (dzoto), capable par conséquent de «nouer le feu » (dzosasà) et de «nouer la parole» (gbesa) lors d'une conjuration (dzoduamé, « le feu mange l'individu ») accompagnée de la manipulation d'amulettes (dzokà, « liane de feu »).

Chez le transgresseur ou entre les mains d'un individu malfaisant, le feu devient un pur agent de mort et de destruction. La faute comme la sorcellerie non seulement compromettent l'ordre établi mais, plus encore, elles rappellent, comme le dit G. Balandier, « la présence active d'un hasard aveugle et la présence d'un désordre irréductible $»^{73}$. Dans les circonstances les plus anodines ou dans celles où la vie est directement menacée, le feu affecte à tout instant la vie des hommes.

\section{L'IRRÉDUCTIBLE INCERTITUDE}

Du dynamisme résultant de l'omniprésence du feu, il ressort que la perspective d'un déséquilibre éventuel conditionne, a priori pour une part non négligeable, la décision d'agir dans un sens plutôt qu'un autre. Mais il arrive aussi à l'homme de ne plus maitriser ses pulsions, au risque de déclencher des feux magiques contre ses semblables d'abord puis contre sa propre personne par retour ensuite. Cette liberté les Ewé la désignent sous le nom de Legba (Legba également chez les Fon, Esu Elegbara chez les Yoruba) ${ }^{74}$, un vodu ayant la particularité de se présenter sous une forme générale et d'être également, sous sa forme privée, à la fois une représentation et une réalisation de chaque homme au «destin » $(s e)$ duquel il préside.

73. Op. cit., p. 122, 1988.

74. Si l'on en croit B. Maupoil (1943, p. 80), Legba serait originaire du pays yoruba et désignerait «celui qui prend le sacrifice». P. Verger (1957, p. 91) précise qu'il faut lui sacrifier en premier, sous peine de réveiller son agressivité naturelle et de le pousser davantage encore à la malveillance. 
Passons sur l'abondante littérature à propos de son rang au sein du panthéon ${ }^{75}$ et abordons de suite la question de sa personnalité. Legba se laisse difficilement cerner tant il déroute. Aussi insaisissable que le feu et le vent auxquels on le compare - et dont on veut lui voir tenir le rôle de « représentant », sans toutefois y voir à proprement parler un dieu du feu ou du vent - il symbolise la part d'imprévisible de l'individu ; la possibilité pour chacun de ne pas être totalement prisonnier de sa condition (sociale, politique...). Symbole de la liberté, il pousse celle-ci à son paroxysme en bafouant les lois et les convenances; son caractère truculent, susceptible, vaniteux, rusé, violent et sans vergogne, déjà repérable à travers des organes reproducteurs monstrueux ${ }^{76}$, fait de lui l'archétype du transgresseur. Cette attitude volontiers iconoclaste, presque folle, s'oppose à l'ordre social et universel, elle s'ingénie à montrer que ce dernier étant inévitablement porteur de désordre, il est par conséquent aléatoire. Au-dessus des pouvoirs (y compris celui de Mawu ! ${ }^{77}$ ), Legba les interpelle sans la moindre retenue, n'hésitant pas à les prendre pour cible directe de son ironie mordante ${ }^{78}$. Toujours en mouvement, il fait rire et pleurer le monde entier ${ }^{79}$. Hors norme, opposant la démesure à la raison, il soutient la comparaison avec des personnages mythiques de l'Égypte pharaonique comme Seth ou du monde grec antique comme Dionysos ou encore, curieusement, Hermès. Car il n'a pas que des mauvais côtés : dans certaines circonstances, on lui prête de traduire sa puissance - son ubris eussent dit les Grecs - dans un sens positif en assurant la protection des carrefours, des marchés, des portails... contre les puissances maléfiques ${ }^{80}$, comme de tenir le rôle d'émissaire auprès

75. L. Frobenius, op. cit. ; B. Maupoil, op. cit.; P. F. Verger, op. cit., 1957 ; H. Courlander, op. cit.

76. P. F. Verger, op. cit., p. 115, 1957.

77. En tant qu'il n'est pas soumis à l'autorité de Mawu Legba ne se distingue pas des autres vodu. Ce qui le caractérise est son irrévérence et le fait qu'il ne jouirait pas, à l'instar de chacun de ses pairs, du pouvoir d'intervenir sur un secteur particulier de la création mais de celui de s'immiscer dans tous, sans distinction, au gré de sa fantaisie.

78. G. Balandier, op. cit., p. 93-96, 1985.

79. B. Maupoil, op. cit., p. 80.

80. Idem, p. $79 \& 82$. 
des hommes et de médiateur parmi les esprits ${ }^{81}$. Ainsi, à condition naturellement de recevoir des offrandes, il balise et protège les parcours individuels et les lieux collectifs.

Si tel le feu qu'il symbolise à l'échelle humaine Legba est ni bon ni mauvais quant à sa nature profonde, il est en revanche l'entité «bonne-mauvaise » au regard de ses manifestations. Vodu de la transgression et du mouvement (donc à la fois partout et nulle part), une irréductible incertitude s'y rattache. De là son pouvoir d'éviter à l'individu la clôture sur lui-même. Pareille aptitude s'enracine comme pour n'importe quel génie dans la puissance d'une « force », comparable, nous dit M. Augé, à « celle de l'énergie atomique qui n'est par elle-même ni bonne ni mauvaise $[\ldots] »^{82}$. On ne saurait trouver meilleure illustration du feu magique dérivé du feu physique. Auparavant, B. Maupoil avait relevé dans un mythe fon la présence d'un feu recouvrant (imprégnant serait plus exacte) le corps d'un Legba au visage ensuite surmonté de flammes ${ }^{83}$. Il n'y a là rien d'étrange si l'on se souvient du rôle prêté au feu dans la dynamique du monde, visible et invisible, ainsi que dans l'émergence du désordre qu'il incarne de façon générale ; permettant de la sorte d'établir une correspondance avec les explosions de violence de Legba - celles de l'homme pour qui celui-ci est une effigie de sa propre colère qu'il s'efforce d'apaiser, nous précise B. Maupoil ${ }^{84}$. Ce vodu incarne la propension de l'individu à créer indifféremment de l'ordre ou du désordre. Selon son usage de la parcelle de feu cosmique enfouie au plus profond de son être, il s'inscrit de diverses manières dans la dynamique d'un univers en perpétuel devenir. Au plan social, l'emploi de ce feu personnel exprimé à travers Legba se traduit dans l'aventure du rapport à l'autre, confondue avec l'expérience intime du rapport à soi-même. L'ambiguïté originelle du couple feu-Legba prenant la forme de l'ambivalence au niveau de l'homme, elle rend par conséquent possible le rejet d'une conception fataliste de l'existence.

81. M. Augé, op. cit., p. 120, 1982.

82. Op. cit., p. 23, 1988.

83. Op. cit., p. 336, 535.

84. Idem, p. 82. 
Malgré les multiples contraintes sociales visant à éviter les écarts à la norme communément admise, subsiste une part d'incertitude quant à la conformité des comportements humains. À cause de son appartenance à l'univers, tout homme dispose déjà de cette énergie qui lui permet d'intervenir sur son parcours terrestre. Legba incarne ce feu, synonyme de liberté mais aussi symbole du danger s'il n'est pas mobilisé à bon escient. Les Ewé tiennent là un discours d'une incontestable portée philosophique : quelle que soit la volonté d'établir un contrôle sans faille, il subsiste toujours en toute chose une part de non mesurable. L'homme n'échappe pas à la règle et, pour ce motif, il risque fort, s'il n'y prend garde, d'être le premier responsable de son propre malheur.

\section{LA FUREUR DES ESPRITS}

Les Ewé axent explicitement leurs analyses sur des états thermiques, réels ou symboliques. Exact contrepoint de la catégorie de désordre associée à la chaleur, on relève la catégorie d'ordre associée à la fraîcheur ; aux humeurs dévastatrices des génies et des vodu, répondent le calme et la douceur de Afà (Fa chez les Fon, Ifa chez les Yoruba), entité spirituelle de la divination, qui se contente de dévoiler aux hommes les voies à suivre (en priorité leurs " destins », se) pour voir la paix enfin régner sur le monde : Fà gbede xosu («Fà est le roi de la vie d'ici-bas ») ${ }^{85}$. Synonyme de vérité, de sagesse et de bonté désintéressée, Afà recouvre l'ensemble des thèmes relatifs à la plénitude physique et morale : fà (« être doux », «s'adoucir», «se radoucir», « être frais »...et « être passif », par opposition à être agressif), fà àko $n a$ (« tranquilliser »), fà do $n a$ ( «soulager»), fàfa ( « refroidissement», « soulagement»), fàfà ( « fraîcheur », « frais », « paisible ») et fàfée («froid »). À l'opposé, nous en avons déjà eu un très large aperçu, aux feux des puissances surnaturelles correspond une multitude de maux affectant l'individu ou le groupe : maladies, sortilèges, zizanie, transgressions du code

85. Ibid, p. 7. 
moral... Cependant il existe des moyens pour mettre un terme au malheur, voire, si nécessaire, pour tenter de s'en prémunir.

L'ensemble les esprits (y compris Afà) se montrent sensibles aux attentions qu'on leur porte ; comme aux négligences dont ils estiment pâtir d'ailleurs. D'où la nécessité de leur dédier des offrandes ; ce qui ne dispense pas d'adopter des attitudes conformes aux prescriptions de l'ordre moral dont ils sont les gardiens les plus efficaces, naturellement. Les rituels sont exécutés selon un schéma de type don-contre-don : en signe de reconnaissance pour les soins prodigués, les hommes espèrent bien voir les esprits ne pas rester insensibles. Il s'agit effectivement là d'un souhait, puisqu'il ne saurait être un seul instant question de les soumettre. Mais il est clair que l'entreprise n'a pas la moindre chance d'aboutir si l'on n'offre pas déjà des «nourritures » appropriées ${ }^{86}$, c'est-à-dire utiles au maintien de la puissance active de l'entité concernée, sous peine de la voir perdre la capacité de mobiliser son feu ${ }^{87}$. Hormis verser du sang (l'esprit se nourrirait du yệ (l' " âme ») de la victime (poulet, bouc...)), le sacrifiant doit conjointement verser des offrandes au nombre desquelles d'indispensables préparations composées de feuilles déchirées ou froissées dans de l'eau, des àmàtsi (« eau d'àmà »). En fonction de l'objectif visé, il emploie soit des «plantes chaudes » (àmà dzodzo) soit des « plantes froides » (àmà fàfà) : les premières encourageront la colère ou l'ardeur combative de l'esprit concerné, les secondes l'apaiseront ou le maintiendront dans une atmosphère de repos. Plusieurs cas de figure sont envisageables. Si le rituel consiste en un processus curatif après l'attaque d'une puissance surnaturelle, les àmà dzodzo auront alors pour but d'exciter l'esprit agressif avant de s'en débarrasser à l'aide (en sus des formules incantatoires de circonstance) d'une àmàtsi de type fàfà.

86. A. de Surgy (op. cit., p. 96, 1995) propose un éclairage sur le mode d'alimentation des esprits : "Il semble donc émaner de la matière en général, et des objets en particulier (c'est-à-dire des portions de matière portant trace d'activité spirituelle), des effluves dont les esprits viennent s'emparer et dont en quelque sorte ils se nourrissent. »

87. Les hommes ont besoin des vodu ; et inversement. Pour ces raisons, on n'hésite pas à suspendre les sacrifices à destination d'un vodu qui a cessé de « travailler ». 
Dans le cas d'une entreprise visant à nuire, le sorcier utilise des àmà $d z o d z o$ afin d'affaiblir le feu de l'esprit dit protecteur de sa victime. Une àmàtsi dzodzo a tout autant le pouvoir d'assurer la protection du sujet par une divinité particulière ou de renforcer sa résistance aux attaques des feux magiques (par exemple ceux des esprits errants). L'emploi d'une àmàtsi fàfà permet de bénéficier de l'énergie spirituelle ou de la chance dispensée par une divinité, ou encore d'un soutien lors d'une période de convalescence. Bien sûr, chaleur et fraîcheur étant toute relative, la qualité $d z o d z o$ ou fàfà d'une essence dépend de la composition dans laquelle elle entre, en vue de conduire au résultat attendu: dans une situation précise, une plante dite « chaude » peut fort bien devenir « froide » par comparaison avec une autre. Néanmoins, quelle que soit l'orientation choisie, l'usage des àmàtsi entend toujours influer sur l'état thermique de l'esprit - le reflet de son humeur - puis, par contrecoup, sur celui du sujet.

L'emploi des àmà revêt une importance capitale. Leur absence du rituel rendrait celui-ci parfaitement inutile; car de leur choix dépendent dans une très large mesure les événements à venir. Chaque esprit réagissant à des plantes spécifiques, il est ainsi conduit à s'activer dans un sens prévisible. Étant entendu que les processus mobilisés valent pour les autres puissances, reprenons l'exemple de Legba en précisant d'abord son aspect matériel. Legba est d'ordinaire représenté par un monticule de terre au sommet duquel apparaît une tête de forme humaine grossièrement stylisée. À l'intérieur de l'effigie, plus précisément à sa base, est dissimulé ce qu'on pourrait appeler sa «formule secrète »; un composé de plusieurs matières (organiques, minérales et animales) dont, parmi elles, toujours une plante supposée conférer son pouvoir magique à l'ensemble. Legba possède sa plante propre à l'instar de n'importe quelle divinité - une conception à ce point fondamentale chez les populations du Golfe de Guinée, que les esclaves lui firent jadis franchir l'Atlantique ${ }^{88}$. En fonction de son objectif, outre les nourritures supposées l'exciter ou le calmer ${ }^{89}$, le sacrifiant recherche les àmà capables de l'activer au maximum de son potentiel. Elles interféreraient positivement ou

88. Lydia Cabrera, El Monte, La Habana, p. 100, 1954.

89. P. F. Verger, op. cit., p. 115, 1957. 
négativement avec la plante enfouie dans l'effigie. Il est communément admis que, par exemple, le versement d'une àmàtsi sous forme d'huile de palme salée, dzomi (« huile de feu»), en citant le nom de la personne à atteindre, déclenchera sa fureur. B. Maupoil énonce le sens de la manipulation de la manière suivante : « $[\ldots]$ ceux qui veulent enflammer leur Legba contre quelqu'un emploient cette méthode ${ }^{90}$. On ne saurait être plus clair sur le rôle « effectif » du feu. M. Augé poursuivra dans le même sens : les « dieux-objets » sont «manipulables, comme peut l'être l'énergie électrique ou atomique $»^{91}$. La comparaison est particulièrement bien choisie, puisque le feu magique envahirait - « dévorerait », faudrait-il dire aussi sûrement qu'un courant ou une radiation.

Que seraient les esprits sans le feu ? Les Ewé en sont convaincus, de pures abstractions. D'ailleurs, peut-être faut-il entrevoir dans leur capacité à mobiliser l'agent destructeur par excellence la première raison pour laquelle ils inspirent un si profond respect. L'éventualité du châtiment incite sans doute à la prudence ; ce qui ne signifie pas qu'on renonce à les manipuler ou à courir le risque de les réveiller. Dans les deux cas cela revient pourtant à déclencher des réactions en chaîne difficilement maîtrisables, voire hors de tout contrôle ; surtout si l'initiative de leur déclenchement revient au surnaturel par réaction à des transgressions d'interdits. Ils suscitent alors d'autant plus l'effroi qu'ils signalent au groupe une menace imminente contre son intégrité.

\section{LA LUTTE CONTRE LA MORT}

Le feu magique jouant un rôle considérable au niveau du social, nous devons maintenant procéder à un examen des conduites transgressives; plus exactement de leurs suites. Quiconque imagine pouvoir ignorer la Loi encourt une sanction et, souvent, à cause du souci du coupable de dissimuler son forfait, une puissance invisible se charge de dévoiler la faute ; une mission accomplie grâce à la mobilisation, une fois encore, d'un feu magique. Le plus «piquant»

90. Op. cit., p. 83.

91. Op. cit., p. 94, 1988. 
est, dit-on, celui de Gu (Egu chez les Fon et Ogun chez les Yoruba), vodu dont aucune parade ne parvient à éviter l'attaque. Il est néanmoins possible de procéder à des réparations en intervenant sur le chaud et sur le froid au cours de rituels appropriés.

Les excès de feu entraînant des ruptures dommageables, l'adoption d'attitudes respectueuses des lois relève de l'impératif : il faut combattre le désordre inhérent à toute organisation humaine. Parce qu'elle concerne la base de la structure sociale, la lutte contre la menace $\mathrm{d}^{\prime}$ ' entropie $»^{92}$ prend un sens aigu à propos des relations hommes-femmes. Des interdits majeurs les balisent sous peine que la terre et les êtres la peuplant ne soient frappés de stérilité. Ici comme ailleurs, celui de l'inceste est le plus grave. Le transgresser signifie s'abandonner à son propre feu, son désir et, de fait, devenir réceptif au feu de Gu qui vient alors le renforcer. L'intervention de ce vodu (le plus entreprenant des vodu terrestres) n'a rien de surprenante, sa résidence habituelle étant le fer, matière-d'œuvre du forgeron à qui il revient de tenir le rôle de dépositaire de l'éthique collective ; une charge mise en parallèle, rappelons-le, avec son « travail » du feu. L'énoncé : « La forge aura de l'avenir tant qu'il y aura des hommes » (Esi wonyé amewo li ta agbédè dó le $\underline{\text { dugo }}$ yige kokoko) le précise : il ne saurait exister de communauté humaine - donc de forge pour en signifier les conditions d'existence - sans l'observation d'un minimum de règles au nombre desquelles, tout d'abord, celle de l'exogamie lignagère. Puisqu'elle nie « la prééminence du social sur le naturel, du collectif sur l'individuel, de l'organisation sur l'arbitraire », nous dit C. Levi-Strauss ${ }^{93}$, l'inceste provoque ici le déchaînement du feu du vodu. Il en va de même pour l'adultère et la violence conjugale : le premier revient à «poser le pied dans la brousse », à retourner vers où se déchaînent les puissances enfiévrées de la nature et la seconde nie, explicitement, que la Loi et donc l'ordre se disent d'abord par le sexe ; de façon générale par un ensemble de relations codifiées entre hommes et femmes.

92. G. Balandier, Anthropo-logiques, Paris, Librairie générale française, p. 238, 1974.

93. C. Lévi-Strauss, Les structures élémentaires de la parenté, ParisLa Haye, Mouton \& Co, p. 52, 1947. 
Leur transgression conduirait, affirme-t-on, à un chaos irréversible en l'absence de rituels réparateurs. Le couple se verrait frappé de stérilité soit parce qu'il refuserait les règles de l'alliance soit parce qu'il compromettrait sa capacité de reproduction. En sus d'inévitables sanctions, le rappel des grands principes passe par une intervention sur le feu. La faute dénoncée par Gu ayant retenu l'attention, on intime alors à celui-ci de cesser de se manifester : «Le feu de Gu est ici! Gu doit sortir!» (Gu dzo le afisià ! Gu dho là tô !). La première incantation mentionne sans équivoque l'importance du feu dans l'intervention du vodu; on lui doit d'avoir permis de dénoncer les fautifs. Après un traitement adéquat de ces derniers à l'aide de bains successifs à base de «plantes chaudes » (àmà dzodzo) puis de «plantes froides » (àmà fàfà), le retour à la normale résulte du rétablissement de l'équilibre énergétique au niveau de chaque partie concernée ${ }^{94}$. Dans le cas d'un adultère une étape supplémentaire intervient: l'amant et le mari doivent se soumettre à une cérémonie appelée dzodhùdhù (littéralement «manger le feu»), jurant par ce geste solennel de désormais ne plus chercher à se nuire d'une quelconque manière. Si l'un des deux revenait sur son engagement, alors le feu ingéré - un feu magique contenu dans un breuvage où a été immergée une pointe de fer symbole de $\mathrm{Gu}$ - se retournerait contre lui et le tuerait ${ }^{95}$. Au-delà d'une simple question

94. Des deux parties la victime est celle qui courrait le plus grand danger puisque, n'ayant pas été l'initiatrice de la faute, elle reçoit de façon inattendue le feu du vodu.

95. A. de Surgy (op. cit., p. 78, 1995) veut voir dans ce type de processus un moyen de capter une parcelle de l'énergie de la puissance surnaturelle concernée : «Au terme de sa cure, l'individu n'est pas simplement guéri mais enrichi par mise en rapport avec la puissance directrice efficace de la divinité ayant été mise en cause. Elle peut en outre utiliser avantageusement sans danger, au lieu de s'en séparer, l'énergie qui était attachée à la formation psychique (personnelle ou extérieure) incriminée. » Si un lien se tisse effectivement entre le vodu et l'individu - s'il s'agit d'une affinité élective, elle se traduit d'ordinaire par la naissance d'une relation de type maître-serviteur et l'individu s'engage désormais à servir le vodu en observant les divers interdits et en lui rendant un culte, ce dernier n'est pas pour autant investi d'une quelconque parcelle de son pouvoir. Au contraire le simple fait d'avoir été à son contact lui intime la prudence, le vodu n'hésitant pas à activer le feu qu'il a déposé pour le rappeler à l'ordre. 
d'honneur ou de l'effacement d'une souillure ${ }^{96}$, ce sont des suites considérablement plus graves qui sont prises en considération. Elles ne concernent plus l'individu, mais le groupe dans son ensemble; la perte de la fertilité et la mort pour faute sont deux événements inducteurs de déséquilibres sociaux : l'un parce qu'il perturbe l'alliance - et par conséquent la circulation des biens fonciers - l'autre parce que, dévoilant le scandale, il génère d'inévitables tensions.

Fondamentalement, la perpétuation de la vie repose sur l'observation d'une éthique rapportée à un jeu sur le chaud et le froid; si trop de feu annonce une offensive du désordre, en revanche l'absence d'un minimum de feu annonce la victoire définitive de la mort (phase ultime et irréversible de l'expression du désordre) sur la vie. Les Ewé ont une conception philosophique de l'homme qui tranche radicalement avec celle d'ordinaire admise : l'être humain n'est pas condamné à subir sans cesse les influences de l'invisible, il doit d'abord s'efforcer de contenir ses pulsions destructrices incarnées symboliquement dans son propre feu. Et il revient aux grands vodu, et au premier chef à $\mathrm{Gu}$, de préserver, si nécessaire, l'ordre moral au moyen de leurs feux.

\section{LE DÉSORDRE INSIDIEUX}

Ce qui motive l'intervention, par définition brutale, des esprits tient souvent - il ne faut pas négliger les assauts imprévisibles au caractère antisocial de diverses attitudes. À l'exception notoire du forgeron, répétons-le, certains individus éveillent naturellement la suspicion à cause de leur contact régulier avec le feu ou bien à cause de leur mode de vie. D'où un ensemble de pratiques magiques visant à maîtriser les débordements d'énergie toujours dommageables, tôt ou tard, au devenir du groupe. Elles se veulent capables de produire les effets attendus, d'être à l'instar des techniques des actions « efficaces ${ }^{97}$.

96. C. Rivière, op. cit., p. 103, 1981.

97. M. Mauss, «Les techniques et la technologie », Journal de Psychologie normale et pathologique, p. 71-78, 1941. 
Les Ewé insistent sur la distinction entre «bons feux »( $d z o$ nyui) et «mauvais feux » (dzo vô), les premiers ayant trait à la magie dite blanche et les seconds à celle dite noire. Cette dernière fait peser une lourde menace sur le lien social. Pourtant la manipulation du feu n'a de prime abord rien de répréhensible - dès lors qu'elle se produit au vu et au su de tous, ni les amulettes par elles-mêmes. Tenons-en une nouvelle fois pour preuve la place du forgeron au sein du groupe : grand maître du feu par excellence, la pureté prêtée à ses intentions lui permet de n'être assujetti ni à l'endogamie ni à un quelconque marquage social dégradant, au contraire ${ }^{98}$. Tel n'est pas le cas de la femme - elle aussi grande manipulatrice du feu facilement accusée de commercer avec des puissances dangereuses. En premier lieu l'ambivalence de sa physiologie rapportée à des influences tantôt régénératrices tantôt dangereuses joue en sa défaveur : durant la période des règles, ses actes et les lieux où elle se rend est gù (« porte-malheur ») pour son entourage immédiat ${ }^{99}$ et, par extension, pour l'ensemble de la communauté villageoise ${ }^{100}$. Cela ne différerait guère des observations habituelles en Afrique 101 si le gù d'ici n'était rien d'autre qu'une référence explicite au vodu $\mathrm{Gu}$, dont on sait que son feu se manifeste de manière systématique pour signaler un désordre; en l'occurrence ici une absence de fécondation pensée symboliquement comme tel. Le sexe féminin assimilable à un four, un thème universel ${ }^{102}$, et la chaleur supposée de la féminité attesteraient autant de prédispositions « naturelles » à la sorcellerie. Ajoutons à cela, facteur aggravant, le contact régulier avec le feu qui désigne la femme - son statut social l'y condamne

98. Formellement, un forgeron ne saurait nourrir de funestes projets sans prendre le risque d'être immédiatement terrassé par le vodu Gu résidant, rappelons-le, dans le fer.

99. J. Spieth, op. cit., Ch. III, p. 14, 1906.

100. Von P. Anton Witte, «Menstruation und Pubertätsfbeier der Mädchen im pandugbiet Togo », Baessler Arch, Beiträge zur völkerkunde, 1, p. 279-280, 1911. P-E. Breitkopf, «Beitrage ur Ethnographie der Kpandoleute », Anthropos, 22 (3-4), p. 477-506, 1927.

101. Luc de Heusch, Le sacrifice dans les religions africaines, Paris, Gallimard, p. 202-251, 1986.

102. James Georges Frazer, The Golden Bough. A Study in Magic and Religion, London, Mc Millan, p. 31, p. 143-144, 1915. 
sans appel - comme particulièrement perméable aux entités transitant entre le visible et l'invisible : la «chaleur» $($ dzodzo $)$ du «foyer» (dzokpo), la «fumée » ou la «vapeur» (adzudzo) et la «cendre » (dzowo ou dzomèfí) renvoient symboliquement à une éventuelle victoire des puissances du désordre. Du feu de son corps, comparable à celui du chaman ${ }^{103}$, naît la vie ; par sa manipulation du feu les aliments passent du cru au cuit, domaine de la culture. En elle s'exprime pêle-mêle l'union des contraires, la vie et la mort ${ }^{104}$. Au vu de ces indices, on la soupçonne toujours de pencher plutôt vers la nature que vers la culture ; une inclination prêtée également à tous les individus qui adoptent des attitudes ou un mode de vie ne recevant pas d'explication immédiate: les riches ou ceux ayant choisi de vivre d'une façon trop modeste pour ne pas être soupçonnés d'avoir pactisé avec des puissances nuisibles. Leur désir de nuire s'exprime, dit-on, à travers des malédictions.

Ce sont les paroles accompagnatrices de l'action sorcière qui suscitent le principal désaveu, dans la mesure où elles empruntent explicitement le feu comme vecteur de la subjectivité. Un proverbe traduit fort bien leur implication: "C'est sur la liane de feu que marche la parole » (Kaji kaji nyaà dzokà). Porteurs d'un message, les mots animeraient le feu dans le sens souhaité. Premier génie domestiqué par les hommes, le verbe est puissance; il implique l'action. Potentiellement dangereuse, la parole est un outil qu'il importe de maîtriser : «La langue c'est la mort » (Adea kùé). Comme dans d'autres contextes culturels, on reconnaît le lien unissant le verbe et son pouvoir de destruction ${ }^{105}$. Pour les Ewé, il faut imputer au verbe chargé de l'intention maléfique le pouvoir d'exploiter la face sombre du feu. Dans le souci de s'en protéger, l'acquisition d'un « charme » $(b \hat{o})$ « chargé » d'un « bon feu », ou l'installation d'un vodu personnel contenant divers ingrédients (débris végétaux,

103. Mircea Eliade, Le chamanisme et les techniques archaïques de l'extase, Paris, Payot, 1954 ; Mythes, rêves et mystères, Paris, Gallimard, 1957.

104. Louis-Vincent Thomas, La mort africaine. Idéologie funéraire en Afrique noire, Paris, Payot, p. 63-64, 1982.

105. Jeanne Favret-Saada, Les mots, la mort, les sorts, Paris, Gallimard, p. 25-30, 192-196, 1977. 
minéraux ou organiques), constituent des mesures efficaces. Censés abriter une « formule concrète d'énergie vitale » ${ }^{106}$, ils favorisent la guérison et éloignent les mauvais esprits. Leurs feux jouent le rôle de « contre-feux » et ce qu' on entend habituellement par «protection» ne désigne en fait rien d'autre que ce dépôt singulier d'énergie cosmique, dont il est indispensable de maintenir l'efficacité au prix d'offrandes régulières. La puissance du charme (ou du vodu) s'oppose à celle du sorcier ; l'intensité de son feu la contrarie au point d'en annuler les effets. L'entreprise sorcière et la lutte qu'elle suscite en retour illustrent cette oscillation permanente entre les risques de déstabilisation et la recherche ininterrompue de formes stables. L'ordre parmi les hommes n'étant ni acquis d'avance ni envisageable de manière définitive, l'équilibre social est inévitablement précaire. Telle est l'idée centrale. Sans un effort régulier pour contenir la menace de feux intempestifs, bref sans une indispensable vigilance visant à repousser le spectre du chaos, l'existence même du groupe relèverait à coup sûr de l'utopie.

\section{LA VIOLENCE}

À l'instar de n'importe quelle société, les Ewé disposent d'une «cartographie de l'ordre et du désordre » et «l'ordre n'a de sens que par comparaison avec ce qui s'en démarque », pour reprendre les formules de G. Balandier ${ }^{107}$. À tout moment la violence risque de surgir, entrainant alors d'inévitables ruptures. Sous la forme de l'agression magique ou de l'agression physique (incluant l'invective), son déchaînement se rapporte toujours à des débordements d'énergie impliquant le surnaturel. Toutefois, et il faut insister sur ce point, les Ewé n'entendent pas imputer aux esprits la responsabilité de tous les événements malheureux affectant le cours de la vie. Si l'invisible est impliqué, il l'est au titre d'un double personnalisé, attestant à travers la mobilisation de son feu magique l'expression d'un désir initial exclusivement humain.

106. A. de Surgy, op. cit., p. 103-143, 1993.

107. Op. cit., p. 100, p. 149-180. 
Le siège de la violence se situe dans l'abdomen, là où se trouve l'esprit imaginant de l'individu ; celui qui dirige les fonctions représentatives et le calcul ${ }^{108}$, en d'autres termes le siège psychologique des sentiments classables en hostiles ou en favorables. La violence est assimilée à Legba, vodu qui, nous l'avons vu, incarne à l'échelle individuelle cette facette de l'être illustrée par le libre jeu des pulsions. On retrouve l'idée chez les Fon : Legba y est « l'agitateur du nombril » ou encore «le chef de la colère », qualités mises directement en rapport avec le nombril où il réside et d'où il insuffle la colère ${ }^{109}$. À ce double de l'homme sont attribuées les causes des maladies, des accidents et des disputes ; un ensemble de situations générées par un excès de feu. "Sont » Legba : l'action de tuer, de blesser, l'accident, la méprise, la colère subite, le lapsus, le cauchemar, les rêves érotiques et la folie passagère ${ }^{110}$. Au feu symbole du geste fou, s'oppose la fraîcheur rapportée à l'exercice de la raison. Par exemple, la « sincérité » ou l' «innocence » se disent domèfàfà (de domè, " ventre », et fàfà, " fraîcheur », littéralement " la fraîcheur du ventre ») et la «colère », domèdzui, forme contractée de domèdzodzo («la chaleur du ventre»). Aux sentiments favorables à la paix et la collaboration correspond la fraîcheur, à ceux source de discorde et de désunion la chaleur. Lors d'une querelle, on affirme que « le feu est entré dans la poche» (dzo dô gamé) de la victime. Cela confirme la grande place reconnue à l'intention. L'expression : «La parole (au sens de la pensée) qui reste dans le ventre n'est pas pure (juste) » (Nyatsidomè menye nyàdzodzoe ô) l'atteste une fois encore, réfutant de la sorte la thèse d'A. de Surgy ${ }^{111}$, selon qui

108. A. de Surgy, op. cit., p. 42, 87, 107, 1988.

109. A. Le Hérissé, op. cit., p. 137.

110. B. Maupoil, op. cit., p. 79-80.

111. A. de Surgy, «Du non-recours des Evhé à la prestation de serment », Droit et Cultures, 17, p. 133-143, 1989. A. de Surgy semblera douter de sa thèse lorsqu'il écrira en 1995 (p. 74) : «Ici comme ailleurs, le désordre ou la perte de contrôle conscient ne témoigne nullement de l'intervention d'agents invisibles ; il se borne à constituer un terrain propice à leur hypothétique intervention. » Faudrait-il alors déduire que « le désordre ou la perte de contrôle », cette foisci « inconscient», pourrait résulter de «l'intervention d'agents invisibles »? Apparemment pas davantage puisque A. de Surgy affirme peu avant (p. 73) : « La torpeur de la conscience ou au contraire son acuité, que se soit sur le plan 
l'individu est davantage agi qu'il n'agit. Ce serait plutôt l'inverse ; et de très loin. Quant aux passages à l'acte à proprement parler, ils représentent un danger considérable ; les coups portés à l'aide d'objets en fer, la condamnation à des besognes dégradantes... signifient expressément une volonté de détruire, puisque la victime « reçoit $\mathrm{Gu} »$ : le feu du vodu l'atteint à son tour par contamination et si aucun rituel réparateur engageant les deux parties n'est entrepris, la mort finit par l'emporter.

S'il n'a pas suivi le conseil de «rafraîchir son cœur », celui animé par un sentiment de colère - « qui a le cœur chaud »-s'expose alors à d'inévitables sanctions; car la sauvegarde du lien social passe par le retour à la paix et à l'équilibre ${ }^{112}$. Il est impossible de dissimuler la faute, a fortiori parce que l'absence de justice condamnerait l'âme de la victime à se transformer en adéla, esprit errant de la brousse. Le coupable est toujours puni afin d'étouffer son feu ravageur; quitte à solliciter les services d'un géomancien (Afà bokono) pour le démasquer au préalable. Outre les fautifs avérés (assassins, voleurs...), de façon générale sont fustigés tous les individus aux comportements contraires aux règles en vigueur. La richesse ostentatoire - rapportée le plus souvent à l'œuvre d'un sorcier, les abus de pouvoir, les manœuvres délictueuses... figurent parmi les principaux dangers que la société villageoise s'efforce au mieux de repousser, au pire de contenir. Signes d'anticonformisme, ils constituent autant de transgressions. Par extension y sont fréquemment associés les modes de vie prenant des libertés avec les valeurs communautaires, tel le mode de vie urbain favorisant l'individualisme, les mœurs de l'élite politico-administrative accusée de jouir de privilèges exorbitants ou encore les éventuelles brutalités du pouvoir, toute chose là aussi versée, très souvent, au compte d'une manipulation de «mauvais feux » $(d z o v \hat{o})$; un mode de lecture

affectif ou cognitif, préserve de toute influence immatérielle indésirable. » Par contre, A. de Surgy (p. 74, note 1) reconnaît bien aux esprits le pouvoir d'intervenir d'une manière positive : "Si un esprit prend la direction d'un système dynamique, ce serait pour y introduire un certain ordre, significatif, et non du chaos, ou du moins pour l'attirer dans une certaine forme de chaos. »

112. J. Spieth, op. cit., p. 422-423, p. 856, 1906 ; op. cit., p. 222, 1911. 
courant en Afrique, afin de conférer du sens à des situations parfois inédites ${ }^{113}$. Installés au sommet de la pyramide sociale, les aînés les dénoncent avec force : leur influence - surtout sur les jeunes, les cadets - sape la cohésion du groupe, elles brisent les réseaux de solidarité et de dépendance constitutifs de l'armature des hiérarchies. Semblable phénomène induit des effets dévastateurs perçus comme des attaques dommageables à la pérennité du groupe ; une autre manière d'aborder la question de la fécondité, cette fois-ci non plus sous un angle moral mais sous celui de la perte du collectif. Le problème éthique ne s'interprète jamais en termes de «bien » ou de « mal », au sens où nous l'entendons d'ordinaire en Occident, il se traduit en termes de « vie » ou de « mort » du groupe.

\section{LE FEU JUSTICIER}

Les Ewé misent en dernière instance sur le feu des vodu pour maintenir l'ordre. Mais jusqu'à quel point ce feu est-il réellement assimilé à une parcelle du feu cosmique ? Ne procédons-nous pas à une lecture abusive, à une surinterprétation, en affirmant y reconnaître le feu sous une forme magique ? Le châtiment infligé à deux catégories de transgresseurs répond à ces questions. On prête au vodu céleste Héviesso la capacité, tel Zeus ou Jupiter, de jeter son feu sur les voleurs et les assassins. Le parallèle établi entre le principe supérieur dont le feu relèverait et la Loi réapparaît très nettement : même s'il n'y a pas de témoin du délit, le coupable est invariablement puni tôt ou tard ${ }^{114}$. Par le truchement de la foudre, l'idée de l'omniscience du feu - une idée largement répandue sur le continent ${ }^{115}$ se profile en filigrane.

L'indispensable force de la Loi impose une référence suprême. Si l'on ne doit jamais exclure d'aménager une disposition, de l'assouplir et pourquoi pas de la refondre, en revanche renoncer à la

113. G. Balandier, Anthropologie politique, Paris, PUF, 1967 ; Sens et puissance. Les dynamiques sociales, Paris, PUF, 1971 ; P. Geshiere, op. cit.

114. P. F. Verger, op. cit., p. 525-537, 1957 ; Bruno Gilli, Héviesso et le bon ordre du monde. Approche d'une religion africaine, Paris, Mémoire EHESS, 1976.

115. D. Zahan, op. cit., p. 74-81. 
justice reviendrait à laisser s'épanouir sans retenue des feux individuels. Aucun acte délictueux ne doit par conséquent échapper à la sanction, y compris celui commis, si possible, à l'abri des regards ni le voleur ni le meurtrier ne souhaitant a priori être reconnus. Ce que l'homme n'a pas vu, Héviesso, lui, le découvrira. D'après la croyance populaire, il accompagne son feu d'une pierre de foudre (sokpe, "pierre de tonnerre »), symbolisée par une hache (sofie, « hache de tonnerre »), pour fendre les criminels à l'instar des arbres. Pareille sanction peut toutefois ne jamais subvenir si le fautif est démasqué à temps. À l'époque pré-coloniale le suspect subissait l'épreuve de l'ordalie. Le symbole du vodu était immergé dans un breuvage ou dans un liquide bouillant. Ingérer la préparation alimentaire - précisons-le, non toxique - puis lécher l'objet en prenant le ciel à témoin signifiaient l'innocence. Par contre le coupable se gardait, paraît-il, de toucher l'un et l'autre. Une réaction identique était observée avec le liquide bouillant. Dans les deux cas passer outre le danger signifiait en effet s'exposer à « la mort de la brousse » (dzogbèkù, de dzogbè, le «feu de la brousse » et de kù, la «mort », soit la mort par le feu de la brousse, synonyme de l'emprise du désordre), espace jonché de périls et où sont de coutume ensevelis tous ceux tués par les puissances surnaturelles. Décédé d'une manière déshonorante, le criminel resterait éternellement à genoux sur des braises de cocotier (le plus ardent des feux) dans le royaume des ancêtres. Finalement, quelle que soit l'épreuve, peu importe le fluide utilisé ; seule l'énergie contenue dans l'objet est supposée intervenir. Le contact avec le fluide puis celui avec le symbole présentent un danger si et seulement si le vodu a de bonnes raisons de s'activer. J. Spieth le note au passage : la crédibilité de la procédure repose sur une mise en scène hautement élaborée et non dénuée de supercheries ${ }^{116}$. Il n'en demeure pas moins que, une seconde fois, l'issue de l'ordalie est traduite sur le mode métaphorique : l'esprit juge par l'intermédiaire de son propre feu, la matière l'abritant et lui-même faisant un en la circonstance. Et uniquement ce feu-là se prononce. Si le coupable échappe parfois au tribunal des hommes, 
il n'a par contre pas la moindre chance de se soustraire à celui de l'invisible.

Faute d'obtenir réparation du préjudice subi devant les autorités locales, le plaignant jouit du droit de solliciter les services d'un prêtre du vodu. Il en attend l'exécution sans délai du rituel utile au lancer de la foudre. Bien qu'il ne soit pas tenu de s'exécuter personne ne peut contrôler les vodu, pas même Mawu, affirme-t-on, l'esprit devrait pourtant ne pas rester sourd à la prière ; a fortiori puisque, outre l'excitation de son feu, le crime est censé lui faire horreur. L'invocation aurait ainsi pour objectif de tenter d'accélérer une procédure de toute façon inéluctable. La sentence qui en découle, la mort sans appel possible, notons-le, illustre le principe même de justice en pays Ewé : quiconque imagine pouvoir agir en marge des règles est indigne d'appartenir au groupe ; devrait-il payer de sa vie cette indignité. Par sa brutalité le châtiment a valeur d'exemple. En assumant la fonction d'ultime rempart contre une toujours possible banalisation du crime, il rend de facto inenvisageable l'impunité. Même si l'avènement d'un ordre définitif relève de l'utopie, le désordre ne saurait pour autant l'emporter: le transgresseur peut certes toujours espérer échapper à la justice des hommes mais pas à celle du ciel, en vertu du principe qu'il ne saurait s'abstraire des contraintes régissant la (bonne) marche du monde sublunaire. Revient ici le thème de l'origine : parce que son mode d'expression en évoque les premiers instants, la foudre de Héviesso a le pouvoir d'ordonner à nouveau le monde. Le ciel peut tout à partir de l'expression de son propre désordre, y compris rendre la justice en rappelant aux criminels la loi de l'univers. Dans le prolongement de ce schéma, le châtiment par la foudre constitue une marque d'infamie : l'âme du coupable devenant une "âme de mauvais mort », elle est interdite d'entrée au royaume des ancêtres. Sauf à vouloir s'affranchir des lois, à vouloir se croire indépendant de ce sur quoi elles s'appuient, en l'occurrence la maîtrise de l'énergie, tout homme doit donc contenir ses pulsions ; il doit fournir une sorte de travail qui requerrait une «énergie spirituelle ${ }^{117}$, ce

117. Op. cit., p. 91, 1995. 
qui revient à mobiliser son propre feu afin de mieux l'apprivoiser. Y compris sous sa forme individuelle le feu joue là encore un rôle capital dans le maintien de la cohésion sociale. Les Ewé ne se soucient donc pas d'asservir les puissances surnaturelles (vaine entreprise au demeurant), ils s'efforcent par contre bien d'intervenir sur la réalité matérielle supposée leur correspondre.

Chez Héviesso le feu magique de l'invisible renoue explicitement avec sa racine physique originelle. Pour « preuve », à qui douterait encore de l'aptitude effective des vodu à « s'enflammer », Héviesso apporte une réponse catégorique. Et cette dernière en renforce une autre cette fois-ci essentielle à un niveau proprement humain : l'ordre social découle d'un principe de justice dont l'expression est déjà inscrite dans l'intimité de l'univers. Ici le chaud et le froid n'affectent pas uniquement la matérialité des êtres et des choses, ils déterminent les supports (voulus comme objectifs) d'une éthique collective indispensable au maintien du lien communautaire.

\section{BILAN DE L'ENQUÊTE}

Sur l'ancienne Côte des Esclaves le feu est l'agent de la dynamique du monde. Grâce à lui le surnaturel s'immisce dans l'espace sublunaire. Il établit de la sorte une médiation entre le visible et l'invisible. Pourtant, malgré son omniprésence dans les discours et les pratiques ainsi que le souci des hommes de l'apprivoiser, on ne saurait soutenir, même implicitement, qu'il fut, avec peut-être d'autres éléments (l'eau, l'air et la terre), l'objet d'une quelconque vénération. Aujourd'hui encore rien ne nous autorise à voir une superposition effective des cultes actuels à des croyances antérieures autour des forces de la nature. À l'appui de cet argument, retenons le caractère profane des éléments, dont celui du plus important d'entre eux, le feu ; corollaire direct d'une nature qui n'est pas pensée telle une discursivité des esprits. Il convient donc de se montrer prudent, en dépit du fait que, sans l'ombre d'un doute, l'observation de phénomènes physiques - thermiques en l'occurrence - constitue manifestement le substrat d'un vaste pan du culturel. 
Ce constat entraîne également un commentaire à propos de ce sur quoi s'appuie l'approche holiste dans ce contexte particulier. Premier point, de très loin majeur, se référer à la nature implique ici l'écoute de ses pulsations. À l'instar de toute chose, l'individu compose avec la dynamique de l'univers. Mais sa condition d'humain lui intime d'éviter que ne se produisent des excès de feu, ces irruptions du désordre promises à d'inévitables sanctions, prononcées puis exécutées par des puissances invisibles le cas échéant. De ce « travail » - déjà sur sa personne - dépend un mode de cohésion du groupe, qui n'est ni donné d'avance ni jamais définitivement acquis non plus. L'inscription de chacun dans l'ordre instauré est la condition sine qua non de sa durée ; car si la nature retrouve tôt ou tard son équilibre global, en revanche le groupe, lui, doit à sa seule vigilance la possibilité de survivre à ses propres crises. Autrement dit, le chaos n'est pas inéluctable.

Enfin, par le prisme d'un élément profane les Ewé livrent une réflexion qui excède la sphère du religieux. Elle l'affirme, l'inévitable incertitude inhérente à la vie ravale au rang d'utopie le projet de tout soumettre à la prévision et à la mesure, bref à la puissance du calcul. Un espace de liberté est inévitablement maintenu ouvert, ce qui intègre de facto le hasard et l'événement à l'échelle humaine. Aucun déterminisme ne cèle le destin : l'avenir de l'homme est entre ses mains. Nous l'avons vu, semblable perspective impose d'évaluer l'action à l'aune de l'éthique collective ; y compris donc la direction du groupe, étant clairement admis que le politique puisse être exposé, lui aussi, à la critique. Quant à savoir si le pouvoir a davantage à craindre des gouvernés que du Ciel, ou inversement, cela nécessiterait l'ouverture d'un nouveau débat.

E-mail : macealain@yahoo.fr 